\title{
Mathematical analysis of a model of chemotaxis arising from morphogenesis
}

\section{Christian Stinner , J. Ignacio Tello and Michael Winkler}

\section{Communicated by H.A. Levine}

We consider non-negative solution couples $(u, v)$ of

$$
\left\{\begin{array}{l}
u_{t}=u_{x x}-\chi\left(\frac{u}{v} v_{x}\right)_{x}-\lambda u \\
v_{t}=1-v+u
\end{array}\right.
$$

with positive parameters $\chi$ and $\lambda$, where the spatial domain is the interval $(0,1)$. This system appears as a limit case of a model for morphogenesis proposed by Bollenbach et al. (Phys. Rev. E. 75, 2007). Under suitable boundary conditions, modeling the presence of a morphogen source at $x=0$, we prove the existence of a global and bounded weak solution using an approximation by problems where diffusion is introduced in the ordinary differential equation. Moreover, we prove the convergence of the solution to the unique steady state provided that $\chi$ is small and $\lambda$ is large enough.

Numerical simulations both illustrate these results and give rise to further conjectures on the solution behavior that go beyond the rigorously proved statements. Copyright $\odot 2012$ John Wiley \& Sons, Ltd.

\section{Introduction}

Chemotaxis is a phenomenon present in many different biological processes where cells and other micro-organisms orient their movement by an external chemical signal, moving towards the direction of the gradient of the concentration of the chemical substance. Some of the biological processes involving chemotactic movement are, for instance, bacteria movement, immune response, vascularization, one of the key processes in tumor growth, wound healing or Morphogenesis. The process of Morphogenesis (the creation, "genesis", of shapes, "morphe") has been studied since the early 20 th century. It occurs in the formation of the embryo and is responsible for the differentiation of the cells to create organs and their organization. During the process, the cell receives a chemical signal the "morphogen" - which binds to the receptors of the cell situated on its surface. The cell monitors the concentration of morphogens received and activates its differentiation. The morphogens are secreted at localized signaling sites, and how they are transported to the cells of the embryo is a controversially discussed question. Theoretical and experimental scientists have considered different possibilities: transport of morphogens by diffusion in the extracellular matrix of the embryo or transport by contact between cells. In the last decade, several authors have considered mathematical models of partial differential equations (see for instance [1-9]) where diffusion is considered totally or partially responsible of the transport. Recently Bollenbach et al. [10] have introduced a mathematical model to describe the transport of morphogens in Epithelia. In [10], a system of PDEs is presented where the transport of morphogens is induced not only by diffusion, but also by the gradient of the concentration of receptors. Chemotactic terms in morphogen transport have been also considered in Merkin et al. [11].

The mathematical model proposed in [10] for the transport in large time scales consists of a system of two equations: A PDE of parabolic type models the time evolution of the total ligand density $u$, and an ODE describes the behavior of the free and bound receptor density $v$. A general form of the spatially one-dimensional version of this model reads

$$
\left\{\begin{array}{l}
u_{t}=\left(\mathrm{d}_{1}(u, v) u_{x}-\mathrm{d}_{2}(u, v) v_{x}\right)_{x}-\kappa_{1}(u, v) u, x \in(0,1), t>0 \\
v_{t}=v_{\mathrm{syn}}(u, v)-\kappa_{2}(u, v) v, x \in(0,1), t>0
\end{array}\right.
$$


where $d_{1}(u, v)$ is the diffusion coefficient, $d_{2}(u, v)$ is the coefficient of morphogen transport induced by the gradient of the receptor concentration, $\kappa_{1}(u, v)$ and $\kappa_{2}(u, v)$ are the degradation rates of the morphogen and receptor, respectively, and $v_{s y n}$ describes the receptor production at the surface of cells. In order to close the problem mathematically, we have to introduce appropriate boundary conditions and initial data. At $x=0$, the flux through the boundary is related to the production rate at the signaling site, that is,

$$
\mathrm{d}_{1}(u, v) \frac{\partial u}{\partial n}-\mathrm{d}_{2}(u, v) \frac{\partial v}{\partial n}=v \text { at } x=0
$$

where $n$ is the outward unit normal. At $x=1$, zero flux boundary conditions are imposed, that is,

$$
\mathrm{d}_{1}(u, v) \frac{\partial u}{\partial n}-\mathrm{d}_{2}(u, v) \frac{\partial v}{\partial n}=0 \text { at } x=1
$$

Bounded non-negative initial data

$$
u(x, 0)=u_{0}(x), v(x, 0)=v_{0}(x)
$$

are assumed to be known.

The coefficients considered in [10] are given by

$$
\mathrm{d}_{1}(u, v):=\frac{D_{1} v}{b_{1} u+b_{2} v}, \mathrm{~d}_{2}(u, v):=\frac{D_{2} u}{b_{1} u+b_{2} v}, \kappa_{2}(u, v):=\frac{\kappa_{3} v-\kappa_{4} u}{v},
$$

and a constant degradation rate $\kappa_{1}$ is assumed there. The receptor production term $v_{\text {syn }}$ is proposed in [10] to be a linear function of the receptors and the blind receptors, that is, $v_{\mathrm{syn}}=a_{0}+a_{1} \underline{v}+a_{2} \bar{v}$, where $\underline{v}$ and $\bar{v}$ are the free and the blind receptor concentration. For further details in the modeling, we refer the reader to [10]. In this paper, we assume that

1. $\underline{v}$ and $\bar{v}$ depend linearly on $u$ and $v$, that is, $v_{\text {syn }}$ is given by $v_{\text {syn }}=\bar{a}_{0}+\bar{a}_{1} v+\bar{a}_{2} u$ with certain constants $\bar{a}_{0}, \bar{a}_{1}$, and $\bar{a}_{2}$.

2. In (1) and (5) we have $b_{1} u<<b_{2} v$.

3. For the constants in (5) and 1 , we have $\bar{a}_{1}<\kappa_{3}$.

After re-normalizing the system and assuming $\frac{D_{1}}{b_{2}\left(\kappa_{3}-\bar{a}_{1}\right)}=1$ and $\bar{a}_{0}=1,(1)$-(4) is simplified to

$$
\left\{\begin{array}{l}
u_{t}=u_{x x}-\chi\left(\frac{u}{v} v_{x}\right)_{x}-\lambda u, x \in(0,1), t>0 \\
v_{t}=1-v+u, x \in(0,1), t>0 \\
u_{x}-\chi \frac{u}{v} v_{x}=-v, x=0, t>0, u_{x}-\chi \frac{u}{v} v_{x}=0, x=1, t>0 \\
u(x, 0)=u_{0}(x), v(x, 0)=v_{0}(x), x \in(0,1)
\end{array}\right.
$$

where $\lambda:=\frac{\kappa_{1}}{\kappa_{3}-\bar{a}_{1}}>0, v>0$, and we assume $\chi \in(0,1)$.

In this paper we, consider the system (6) and study the well-posedness of the problem under mild assumptions on the initial data. The main results of the paper concern global existence and boundedness of weak solutions (see Lemma 2.6 and Lemma 2.7), as well as asymptotic stability of the unique steady state for a range of parameters (see Theorem 4.1). A regularization method is considered to study the problem, where diffusion is introduced in the ODE. When the diffusion coefficient goes to zero the solutions of the approximating problem indeed approach a solution of (6).

Before going into details, let us establish some further connection to the existing literature. We first note that mathematical aspects of morphogenesis models have been considered by several authors. For instance, Merkin et al. [11] have introduced a chemosensitivity term in the model to describe morphogen concentration. They have presented results on the existence and uniqueness of classical and self-similar solutions to the corresponding system. Their numerical simulations have shown periodic pulse solutions. Mathematical analysis of models of morphogenesis has also been presented in Krzyżanowski et al. [4], where convergence of associated solutions to steady states is studied. Krzyżanowski et al. [5] also considered a mathematical model with nonlinear diffusion and without chemotactic term. The authors present their model and study the steady states, numerical simulations show the sensitivity of the solutions with respect to some parameters.

Next, viewing (6) from a mathematical point of view, one observes that as a consequence of the limit procedure included in our modeling, the "chemotactic sensitivity" $\chi(u, v)$, chosen as $\chi(u, v):=\chi \cdot \frac{u}{v}$ in (6), becomes singular at zeros of $v$. Singular chemotactic sensitivity functions of this type have been considered in various models for chemotaxis processes other than morphogen transport (cf. $[12,13]$ and the survey [14], for instance). These and related works seem to indicate that the analysis of chemotaxis models with such sensitivity functions is more delicate than in neighboring regular situations [14-17].

This paper is organized as follows: In Section 1, the approximation procedure is made precise and a concept of weak solutions is introduced. Section 2 is devoted to the derivation of the global existence and boundedness results of the approximate problems. Moreover, in Section 2, we show that the approximating solutions converge to a global and bounded weak solution of (6). In Section 3, the existence of a unique steady state of (6) is asserted. In Section 4, we establish the global attractivity of the steady state for a certain range of the parameters. In order to illustrate the behavior of the solutions for different data and parameters, we present in the last section some numerical experiments. 
Throughout this paper, we define $\Omega:=(0,1)$, assume $\lambda>0$ and $v>0$ and require that the initial data satisfy

$$
u_{0}, v_{0} \in C^{1}(\bar{\Omega}), u_{0} \geq 0 \text {, and } v_{0}>0 \text { in } \bar{\Omega} \text {. }
$$

\section{Preliminaries and approximation of solutions}

In this section, we first introduce the definition of a global weak solution of (6). This weak solution will be obtained through approximation by solutions of certain regularized problems. The existence of classical solutions to the approximate problems will also be part of this section.

Definition 1.1

Let $\chi>0$. Then by a global weak solution of $(6)$, we mean a couple $(u, v)$ of functions

$$
u \in L_{\text {loc }}^{\infty}(\bar{\Omega} \times[0, \infty)) \cap L_{\text {loc }}^{1}\left([0, \infty) ; W^{1,1}(\Omega)\right)
$$

and

$$
v \in L_{\mathrm{loc}}^{\infty}(\bar{\Omega} \times[0, \infty)) \cap L_{\mathrm{loc}}^{\infty}\left([0, \infty) ; W^{1,1}(\Omega)\right),
$$

such that $u \geq 0$ and $v>0$ a.e. in $\Omega \times(0, \infty)$, and such that the identities

$$
\begin{aligned}
-\int_{0}^{\infty} \int_{\Omega} u \varphi_{t}-\int_{\Omega} u_{0}(x) \varphi(x, 0) \mathrm{d} x= & -\int_{0}^{\infty} \int_{\Omega} u_{x} \varphi_{x}+\chi \int_{0}^{\infty} \int_{\Omega} \frac{u}{v} v_{x} \varphi_{x} \\
& +v \int_{0}^{\infty} \varphi(0, t) \mathrm{d} t-\lambda \int_{0}^{\infty} \int_{\Omega} u \varphi
\end{aligned}
$$

and

$$
-\int_{0}^{\infty} \int_{\Omega} v \varphi_{t}-\int_{\Omega} v_{0}(x) \varphi(x, 0) \mathrm{d} x=\int_{0}^{\infty} \int_{\Omega}(1-v+u) \varphi
$$

hold for all $\varphi \in C_{0}^{\infty}(\bar{\Omega} \times[0, \infty))$.

In order to construct a weak solution of (6) in a convenient way, let us consider the parabolic-parabolic regularization of (6) given by

$$
\left\{\begin{array}{l}
u_{\varepsilon t}=u_{\varepsilon x x}-\chi\left(\frac{u_{\varepsilon}}{v_{\varepsilon}} v_{\varepsilon x}\right)_{x}-\lambda u_{\varepsilon}, x \in(0,1), t>0, \\
v_{\varepsilon t}=\varepsilon v_{\varepsilon x x}+1-v_{\varepsilon}+u_{\varepsilon}, x \in(0,1), t>0, \\
u_{\varepsilon x}=-v, v_{\varepsilon x}=0, x=0, t>0, u_{\varepsilon x}=v_{\varepsilon x}=0, x=1, t>0, \\
u_{\varepsilon}(x, 0)=u_{0}(x), \quad v_{\varepsilon}(x, 0)=v_{0}(x), x \in(0,1),
\end{array}\right.
$$

for $\varepsilon \in(0,1)$.

Using arguments similar to those in [18] and [16], we now show the local existence and uniqueness of solutions to (8).

Lemma 1.2

Let $\chi>0, \varepsilon \in(0,1), q>1, u_{0} \in C^{0}(\bar{\Omega})$ and $v_{0} \in W^{1, q}(\Omega)$ such that $(7)$ is fulfilled. Then there exists $T_{\varepsilon} \in(0, \infty]$ and a pair $\left(u_{\varepsilon}, v_{\varepsilon}\right)$ of functions

$$
\begin{aligned}
& u_{\varepsilon} \in C^{0}\left(\left[0, T_{\varepsilon}\right) ; C^{0}(\bar{\Omega})\right) \cap C^{2,1}\left(\bar{\Omega} \times\left(0, T_{\varepsilon}\right)\right), \\
& v_{\varepsilon} \in C^{0}\left(\left[0, T_{\varepsilon}\right) ; C^{0}(\bar{\Omega})\right) \cap L_{\operatorname{loc}}^{\infty}\left(\left[0, T_{\varepsilon}\right) ; W^{1, q}(\Omega)\right) \cap C^{2,1}\left(\bar{\Omega} \times\left(0, T_{\varepsilon}\right)\right),
\end{aligned}
$$

which solves (8) in the classical sense. If $T_{\varepsilon}<\infty$ then

$$
\left\|u_{\varepsilon}(t)\right\|_{L^{\infty}(\Omega)}+\left\|v_{\varepsilon}(t)\right\|_{W^{1, q}(\Omega)} \rightarrow \infty \text { as } t \nearrow T_{\varepsilon} .
$$

Moreover, $\left(u_{\varepsilon}, v_{\varepsilon}\right)$ satisfies

$$
\begin{gathered}
u_{\varepsilon} \geq 0 \text { in } \bar{\Omega} \times\left[0, T_{\varepsilon}\right), \\
v_{\varepsilon} \geq \min \left\{1, \min _{x \in \bar{\Omega}} v_{0}(x)\right\}=: c_{0}>0 \text { in } \bar{\Omega} \times\left[0, T_{\varepsilon}\right), \\
\int_{\Omega} u_{\varepsilon}(x, t) \mathrm{d} x \leq \max \left\{\int_{\Omega} u_{0}, \frac{v}{\lambda}\right\}=: k_{1} \text { for all } t \in\left(0, T_{\varepsilon}\right),
\end{gathered}
$$


and

$$
\int_{\Omega} v_{\varepsilon}(x, t) \mathrm{d} x \leq \max \left\{\int_{\Omega} v_{0}, 1+k_{1}\right\}=: c_{1} \text { for all } t \in\left(0, T_{\varepsilon}\right)
$$

Proof

We fix $\varepsilon \in(0,1)$. In order to cope with the boundary conditions and the singularity in the first equation of $(8)$, we set $\varphi(x):=$ $-v x(1-x)^{2}, x \in \bar{\Omega}$, and choose a nondecreasing function $\rho \in C^{1+\alpha}(\mathbb{R})$ such that

$$
\rho(s)=\left\{\begin{array}{cl}
s & \text { for } s \geq \frac{c_{0}}{2}, \\
\frac{c_{0}}{4} & \text { for } s \leq \frac{c_{0}}{4},
\end{array}\right.
$$

where $\alpha$ is positive and $c_{0}$ is defined in (11). If $\left(u_{\varepsilon}, v_{\varepsilon}\right)$ is a solution of

$$
\left\{\begin{array}{l}
u_{\varepsilon t}=u_{\varepsilon x x}-\chi\left(\frac{u_{\varepsilon}}{\rho\left(v_{\varepsilon}\right)} v_{\varepsilon x}\right)_{x}-\lambda u_{\varepsilon}, x \in(0,1), t>0, \\
v_{\varepsilon t}=\varepsilon v_{\varepsilon x x}+1-v_{\varepsilon}+u_{\varepsilon}, x \in(0,1), t>0, \\
u_{\varepsilon x}=-v_{1}, v_{\varepsilon x}=0, x=0, t>0, u_{\varepsilon x}=v_{\varepsilon x}=0, x=1, t>0, \\
u_{\varepsilon}(x, 0)=u_{0}(x), \quad v_{\varepsilon}(x, 0)=v_{0}(x), x \in(0,1),
\end{array}\right.
$$

then $\left(\tilde{u}_{\varepsilon}, v_{\varepsilon}\right)$, where $\tilde{u}_{\varepsilon}(x, t):=u_{\varepsilon}(x, t)-\varphi(x)$, is a solution of the problem

$$
\left\{\begin{array}{l}
\tilde{u}_{\varepsilon t}=\tilde{u}_{\varepsilon x x}-\chi\left(\frac{\tilde{u}_{\varepsilon}+\varphi}{\rho\left(v_{\varepsilon}\right)} v_{\varepsilon x}\right)_{x}-\lambda\left(\tilde{u}_{\varepsilon}+\varphi\right)+\varphi_{x x}, x \in(0,1), t>0, \\
v_{\varepsilon t}=\varepsilon v_{\varepsilon x x}+1-v_{\varepsilon}+\tilde{u}_{\varepsilon}+\varphi, x \in(0,1), t>0, \\
\tilde{u}_{\varepsilon x}=v_{\varepsilon x}=0, x \in\{0,1\}, t>0, \\
\tilde{u}_{\varepsilon}(x, 0)=u_{0}(x)-\varphi(x, 0)=: \tilde{u}_{0}(x), v_{\varepsilon}(x, 0)=v_{0}(x), x \in(0,1) .
\end{array}\right.
$$

To obtain a solution of the latter problem, we let $B:=\left\{\left(\tilde{u}_{\varepsilon}, v_{\varepsilon}\right) \in X:\left\|\left(\tilde{u}_{\varepsilon}, v_{\varepsilon}\right)\right\|_{X} \leq R\right\}$ denote the closed subset of the space $X:=C^{0}\left([0, T] ; C^{0}(\bar{\Omega})\right) \times L^{\infty}\left([0, T] ; W^{1, q}(\Omega)\right)$ equipped with the norm $\left\|\left(\tilde{u}_{\varepsilon}, v_{\varepsilon}\right)\right\| X:=\left\|\tilde{u}_{\varepsilon}\right\|_{L} \infty(\Omega \times(0, T))+\left\|v_{\varepsilon}\right\|_{L} \infty\left((0, T) ; W^{1, q}(\Omega)\right)$, where $T$ and $R$ are positive constants. Moreover, for $\left(\tilde{u}_{\varepsilon}, v_{\varepsilon}\right) \in B$ and $t \in[0, T]$, we set

$$
\Psi\left(\tilde{u}_{\varepsilon}, v_{\varepsilon}\right)(t):=\left(\begin{array}{c}
e^{t \Delta} \tilde{u}_{0}+\int_{0}^{t} e^{(t-s) \Delta}\left[-\nabla \cdot\left(\chi \frac{\tilde{u}_{\varepsilon}+\varphi}{\rho\left(v_{\varepsilon}\right)} \nabla v_{\varepsilon}\right)-\lambda\left(\tilde{u}_{\varepsilon}+\varphi\right)+\varphi_{X X}\right] \mathrm{d} s \\
e^{t(\varepsilon \Delta-1)} v_{0}+\int_{0}^{t} e^{(t-s)(\varepsilon \Delta-1)}\left(1+\tilde{u}_{\varepsilon}+\varphi\right) \mathrm{d} s
\end{array}\right),
$$

where $\Delta$ denotes the Laplacian in $\Omega$ with homogeneous Neumann boundary conditions. Then, using the estimates on the heat semigroup from [18, Section 2] and the regularity of $\tilde{\chi}(s):=\frac{\chi}{\rho(s)}$, we can prove in a way similar to the proof of [16, Lemma 2.1] that $\Psi$ has a fixed point $\left(\tilde{u}_{\varepsilon}, v_{\varepsilon}\right) \in B$, which has the claimed regularity and solves (15) in the classical sense, if $R$ is chosen large enough and $T$ is fixed small enough, where $T$ can be chosen independent of $\varepsilon \in(0,1)$. Moreover, setting $u_{\varepsilon}:=\tilde{u}_{\varepsilon}+\varphi$, we see that $\left(u_{\varepsilon}, v_{\varepsilon}\right)$ is a classical solution of (14) that has the claimed regularity properties and fulfills (9) with its maximal existence time $T_{\varepsilon} \geq T$ (see [18, Theorem 3.1$]$ ). As $u_{0}$ is non-negative and $\frac{\partial}{\partial n} u_{\varepsilon} \geq 0$ on $\partial \Omega$, the comparison principle applied to the first equation of (14) yields (10). Then, using the second equation in (14), we have $v_{\varepsilon t}-\varepsilon v_{\varepsilon x x} \geq 1-v_{\varepsilon}$ and apply the comparison principle to deduce

$$
v_{\varepsilon}(x, t) \geq\left(\left[\min _{x \in \bar{\Omega}} v_{0}(x)\right]-1\right) e^{-t}+1,(x, t) \in \bar{\Omega} \times\left[0, T_{\varepsilon}\right)
$$

This yields (11) and, hence, $\left(u_{\varepsilon}, v_{\varepsilon}\right)$ is a classical solution of (8) due to the definition of $\rho$. Integration of the first equation of (8) implies

$$
\frac{\mathrm{d}}{\mathrm{d} t} \int_{\Omega} u_{\varepsilon}(x, t) \mathrm{d} x=v-\lambda \int_{\Omega} u_{\varepsilon}(x, t) \mathrm{d} x
$$

and

$$
\int_{\Omega} u_{\varepsilon}(x, t) \mathrm{d} x=\left(\int_{\Omega} u_{0}(x) \mathrm{d} x-\frac{\nu}{\lambda}\right) e^{-\lambda t}+\frac{\nu}{\lambda}, t \in\left[0, T_{\varepsilon}\right)
$$

which ensures (12). Then, integrating the second equation of (8), we obtain

$$
\frac{\mathrm{d}}{\mathrm{d} t} \int_{\Omega} v_{\varepsilon}(x, t) \mathrm{d} x \leq 1+k_{1}-\int_{\Omega} v_{\varepsilon}(x, t) \mathrm{d} x
$$

and by comparison

$$
\int_{\Omega} v_{\varepsilon}(x, t) \mathrm{d} x \leq\left(\int_{\Omega} v_{0}(x) \mathrm{d} x-1-k_{1}\right) e^{-t}+1+k_{1}, t \in\left[0, T_{\varepsilon}\right) .
$$

This verifies (13) and completes the proof. 


\section{Estimates for solutions of (8). Global existence and boundedness}

In this section, we prove the existence of a global and bounded weak solution of (6). The main ingredients of the proof are $\varepsilon$ independent bounds on norms in $L^{p}$ and $W^{1, s}$ of the solutions $\left(u_{\varepsilon}, v_{\varepsilon}\right)$ to $(8)$, where $p \in(1, \infty)$ and $s \in(1,2)$. The boundedness of $(u, v)$ will then be obtained by a Moser-type iteration procedure.

We first prove the following weighted integral estimates that will be crucial to our approach.

Lemma 2.1

Let $\chi \in(0,1)$. Then for all $p>1$ there exist $\Gamma_{p}>0$ and $\varepsilon_{p}:=\min \left\{\frac{2(1-\chi)}{p \chi}, 1\right\} \in(0,1]$ such that

$$
\int_{\Omega}\left(v_{\varepsilon}^{-(p-1) \chi} u_{\varepsilon}^{p}\right)(\cdot, t) \leq \Gamma_{p} \text { for all } t \in\left(0, T_{\varepsilon}\right) \text { and any } \varepsilon \in\left(0, \varepsilon_{p}\right)
$$

and

$$
\int_{t}^{t+1} \int_{\Omega} v_{\varepsilon}^{\chi} \cdot\left(\left(\frac{u_{\varepsilon}}{v_{\varepsilon}^{\chi}}\right)^{\frac{p}{2}}\right)_{\chi}^{2} \leq \Gamma_{p} \text { for all } t \in\left(0, T_{\varepsilon}\right) \text { and any } \varepsilon \in\left(0, \varepsilon_{p}\right)
$$

Proof

We fix $p>1$ and $\varepsilon \in(0,1)$ and define $s:=(p-1) \chi$ and $z_{\varepsilon}:=\frac{u_{\varepsilon}}{v_{\varepsilon}^{\chi}}$. Then for any $t \in\left(0, T_{\varepsilon}\right)$, we compute

$$
\begin{aligned}
\frac{\mathrm{d}}{\mathrm{d} t} \int_{\Omega} v_{\varepsilon}^{-s} u_{\varepsilon}^{p}= & -s \int_{\Omega} v_{\varepsilon}^{-s-1} u_{\varepsilon}^{p}\left(\varepsilon v_{\varepsilon X x}+1-v_{\varepsilon}+u_{\varepsilon}\right) \\
& +p \int_{\Omega} v_{\varepsilon}^{-s} u_{\varepsilon}^{p-1}\left(u_{\varepsilon x}-\chi \frac{u_{\varepsilon}}{v_{\varepsilon}} v_{\varepsilon x}\right)_{x}-p \lambda \int_{\Omega} v_{\varepsilon}^{-s} u_{\varepsilon}^{p} \\
= & -s \varepsilon \int_{\Omega} v_{\varepsilon}^{-s-1} u_{\varepsilon}^{p} v_{\varepsilon x x}-s \int_{\Omega} v_{\varepsilon}^{-s-1} u_{\varepsilon}^{p}\left(1+u_{\varepsilon}\right)+(s-p \lambda) \int_{\Omega} v_{\varepsilon}^{-s} u_{\varepsilon}^{p} \\
& -p(p-1) \int_{\Omega} v_{\varepsilon}^{-s} u_{\varepsilon}^{p-2}\left(u_{\varepsilon x}-\chi \frac{u_{\varepsilon}}{v_{\varepsilon}} v_{\varepsilon x}\right)^{2}+p v\left(v_{\varepsilon}^{-s} u_{\varepsilon}^{p-1}\right)(0, t) \\
= & -s \varepsilon \int_{\Omega} v_{\varepsilon}^{-s-1} u_{\varepsilon}^{p} v_{\varepsilon X x}-s \int_{\Omega} v_{\varepsilon}^{-s-1} u_{\varepsilon}^{p}\left(1+u_{\varepsilon}\right)+(s-p \lambda) \int_{\Omega} v_{\varepsilon}^{-s} u_{\varepsilon}^{p} \\
& -\frac{4(p-1)}{p} \int_{\Omega} v_{\varepsilon}^{\chi}\left(z_{\varepsilon}^{\frac{p}{2}}\right)_{x}^{2}+p v z_{\varepsilon}^{p-1}(0, t) .
\end{aligned}
$$

As the definition of $z_{\varepsilon}$ implies $z_{\varepsilon x}=v_{\varepsilon}^{-\chi}\left(u_{\varepsilon x}-\chi \frac{u_{\varepsilon}}{v_{\varepsilon}} v_{\varepsilon x}\right)$ and $u_{\varepsilon x}=\chi \frac{u_{\varepsilon}}{v_{\varepsilon}} v_{\varepsilon x}+v_{\varepsilon}^{\chi} z_{\varepsilon x}$ due to the choice of $s$ we obtain

$$
\begin{aligned}
-s \varepsilon \int_{\Omega} v_{\varepsilon}^{-s-1} u_{\varepsilon}^{p} v_{\varepsilon X X}= & -s(s+1) \varepsilon \int_{\Omega} v_{\varepsilon}^{-s-2} u_{\varepsilon}^{p} v_{\varepsilon X}^{2}+p s \varepsilon \chi \int_{\Omega} v_{\varepsilon}^{-s-2} u_{\varepsilon}^{p} v_{\varepsilon X}^{2} \\
& +p s \varepsilon \int_{\Omega} v_{\varepsilon}^{\chi-s-1} u_{\varepsilon}^{p-1} z_{\varepsilon X} v_{\varepsilon X} \\
\leq & -s \varepsilon(1-\chi) \int_{\Omega} v_{\varepsilon}^{-s-2} u_{\varepsilon}^{p} v_{\varepsilon X}^{2}+s \varepsilon(1-\chi) \int_{\Omega} v_{\varepsilon}^{-s-2} u_{\varepsilon}^{p} v_{\varepsilon X}^{2} \\
& +\frac{p^{2} s^{2} \varepsilon^{2}}{4 s \varepsilon(1-\chi)} \int_{\Omega} v_{\varepsilon}^{2 \chi-s} u_{\varepsilon}^{p-2} z_{\varepsilon X}^{2} \\
= & \frac{s \varepsilon}{1-\chi} \int_{\Omega} v_{\varepsilon}^{\chi}\left(z_{\varepsilon}^{\frac{p}{2}}\right)_{X}^{2} .
\end{aligned}
$$

Using now $|\Omega|=1$ as well as Hölder's and Young's inequality, we obtain for any $\delta>0$ and any $w \in W^{1,2}(\Omega)$ that

$$
\begin{aligned}
w^{2}(0) & =\int_{\Omega} w^{2}(0) \mathrm{d} x=\int_{\Omega}\left(w^{2}(x)-2 \int_{0}^{x} w(y) w_{x}(y) \mathrm{d} y\right) \mathrm{d} x \\
& \leq\|w\|_{L^{2}(\Omega)}^{2}+2\|w\|_{L^{2}(\Omega)}\left\|w_{x}\right\|_{L^{2}(\Omega)} \leq \delta\left\|w_{x}\right\|_{L^{2}(\Omega)}^{2}+\left(1+\frac{1}{2 \delta}\right)\|w\|_{L^{2}(\Omega)}^{2} .
\end{aligned}
$$

Hence, we deduce from Young's inequality and (11) that

$$
\begin{aligned}
p v z_{\varepsilon}^{p-1}(0, t) & \leq \frac{p-1}{p \cdot p^{\frac{1}{p-1}}}(p v)^{\frac{p}{p-1}} z_{\varepsilon}^{p}(0, t)+1 \\
& \leq(p-1) v^{\frac{p}{p-1}}\left[\delta\left\|\left(z_{\varepsilon}^{\frac{p}{2}}\right)\right\|_{x}^{2} L_{L^{2}(\Omega)}+\left(1+\frac{1}{2 \delta}\right)\left\|z_{\varepsilon}^{\frac{p}{2}}\right\|_{L^{2}(\Omega)}^{2}\right]+1 \\
& \leq(p-1) v^{\frac{p}{p-1}}\left[\delta c_{0}^{-\chi} \int_{\Omega} v_{\varepsilon}^{\chi}\left(z_{\varepsilon}^{\frac{p}{2}}\right)_{x}^{2}+\left(1+\frac{1}{2 \delta}\right) c_{0}^{-\chi} \int_{\Omega} v_{\varepsilon}^{-s} u_{\varepsilon}^{p}\right]+1
\end{aligned}
$$

Choosing now $\delta:=c_{0}^{\chi} \nu^{-\frac{p}{p-1}} p^{-1}$ and recalling that $\varepsilon_{p}=\min \left\{\frac{2(1-\chi)}{p \chi}, 1\right\}$, for any $\varepsilon \in\left(0, \varepsilon_{p}\right)$ we deduce from (21)-(23) that

$$
\begin{aligned}
\frac{\mathrm{d}}{\mathrm{d} t} \int_{\Omega} v_{\varepsilon}^{-s} u_{\varepsilon}^{p} \leq & {\left[(p-1) v^{\frac{p}{p-1}}\left(1+\frac{p v^{\frac{p}{p-1}}}{2 c_{0}^{\chi}}\right) c_{0}^{-\chi}+s-p \lambda\right] \int_{\Omega} v_{\varepsilon}^{-s} u_{\varepsilon}^{p} } \\
& -\frac{(p-1)}{p} \int_{\Omega} v_{\varepsilon}^{\chi}\left(z_{\varepsilon}^{\frac{p}{2}}\right)_{X}^{2}+1 .
\end{aligned}
$$


Hence, there are positive constants $C_{1}$ and $C_{2}$ depending only on $p, \chi, \lambda$, and $v$ such that

$$
\frac{\mathrm{d}}{\mathrm{d} t} \int_{\Omega} v_{\varepsilon}^{-s} u_{\varepsilon}^{p}+C_{1} \int_{\Omega} v_{\varepsilon}^{\chi}\left(\left(\frac{u_{\varepsilon}}{v_{\varepsilon}^{\chi}}\right)^{\frac{p}{2}}\right)_{\chi}^{2} \leq C_{2} \int_{\Omega} v_{\varepsilon}^{-s} u_{\varepsilon}^{p}+1
$$

is valid for any $t \in\left(0, T_{\varepsilon}\right)$ and $\varepsilon \in\left(0, \varepsilon_{p}\right)$.

Using $s=(p-1) \chi$ and writing $w_{\varepsilon}:=\left(\frac{u_{\varepsilon}}{v_{\varepsilon}^{\chi}}\right)^{\frac{p}{2}}$, we thus obtain

$$
\frac{\mathrm{d}}{\mathrm{d} t} \int_{\Omega} v_{\varepsilon}^{\chi} w_{\varepsilon}^{2}+C_{1} \int_{\Omega} v_{\varepsilon}^{\chi} w_{\varepsilon X}^{2} \leq C_{2} \int_{\Omega} v_{\varepsilon}^{\chi} w_{\varepsilon}^{2}+1
$$

for any $t \in\left(0, T_{\varepsilon}\right)$ and $\varepsilon \in\left(0, \varepsilon_{p}\right)$. Because $\chi \in(0,1)$, we may therefore use Hölder's inequality and (13) to estimate

$$
\int_{\Omega} v_{\varepsilon}^{\chi} W_{\varepsilon}^{2} \leq\left(\int_{\Omega} v_{\varepsilon}\right)^{\chi} \cdot\left(\int_{\Omega} w_{\varepsilon}^{\frac{2}{1-\chi}}\right)^{1-\chi} \leq c_{1}^{\chi}\left\|w_{\varepsilon}\right\|_{L^{1-\chi}(\Omega)}^{2} .
$$

Now from (11) and (12), we know that

$$
\left\|w_{\varepsilon}(t)\right\|_{L^{\frac{2}{p}}(\Omega)}=\left(\int_{\Omega} w_{\varepsilon}^{\frac{2}{p}}\right)^{\frac{p}{2}}=\left(\int_{\Omega} v_{\varepsilon}^{-\chi} u_{\varepsilon}\right)^{\frac{p}{2}} \leq\left(c_{0}^{-\chi} k_{1}\right)^{\frac{p}{2}}=: C_{3}
$$

for all $t \in\left(0, T_{\varepsilon}\right)$ and $\varepsilon \in(0,1)$. Consequently, involving the Gagliardo-Nirenberg inequality, it yields

$$
\begin{aligned}
\left\|W_{\varepsilon}(t)\right\|_{L^{\frac{2}{1-\chi}}(\Omega)}^{2} & \leq C_{4}\left(\left\|W_{\varepsilon X}(t)\right\|_{L^{2}(\Omega)}^{2 a} \cdot\left\|w_{\varepsilon}(t)\right\|_{L^{\frac{2}{p}}(\Omega)}^{2(1-a)}+\left\|W_{\varepsilon}(t)\right\|_{L^{\frac{2}{p}}(\Omega)}^{2}\right) \\
& \leq C_{4}\left(\left\|w_{\varepsilon X}(t)\right\|_{L^{2}(\Omega)}^{2 a} \cdot C_{3}^{2(1-a)}+C_{3}^{2}\right)
\end{aligned}
$$

for any $t \in\left(0, T_{\varepsilon}\right)$ and $\varepsilon \in(0,1)$, where

$$
-\frac{1-\chi}{2}=\left(1-\frac{1}{2}\right) a-\frac{p}{2}(1-a)
$$

that is

$$
a=\frac{p-1+\chi}{p+1} \in\left(\frac{p-1}{p+1}, \frac{p}{p+1}\right)
$$

Thus, from (28), we gain

$$
\left\|W_{\varepsilon}(t)\right\|_{L^{\frac{2}{1-\chi}}(\Omega)}^{2} \leq C_{5}\left\|W_{\varepsilon x}(t)\right\|_{L^{2}(\Omega)}^{2 a}+C_{6}
$$

so that (27) shows that

$$
C_{5}\left\|w_{\varepsilon x}\right\|_{L^{2}(\Omega)}^{2 a}+C_{6} \geq c_{1}^{-\chi} \int_{\Omega} v_{\varepsilon}^{\chi} w_{\varepsilon}^{2}
$$

and accordingly

$$
\int_{\Omega} w_{\varepsilon X}^{2} \geq C_{7}\left(\int_{\Omega} v_{\varepsilon}^{\chi} w_{\varepsilon}^{2}\right)^{\frac{1}{a}}-C_{8}
$$

for any $t \in\left(0, T_{\varepsilon}\right)$ and $\varepsilon \in(0,1)$. Using this, along with (11) and (26), we infer that

$$
\frac{\mathrm{d}}{\mathrm{d} t} \int_{\Omega} v_{\varepsilon}^{\chi} w_{\varepsilon}^{2}+\frac{C_{1}}{2} \int_{\Omega} v_{\varepsilon}^{\chi} w_{\varepsilon x}^{2} \leq C_{9}+C_{2} \int_{\Omega} v_{\varepsilon}^{\chi} w_{\varepsilon}^{2}-C_{10}\left(\int_{\Omega} v_{\varepsilon}^{\chi} w_{\varepsilon}^{2}\right)^{\frac{1}{a}}
$$

for any $t \in\left(0, T_{\varepsilon}\right)$ and $\varepsilon \in\left(0, \varepsilon_{p}\right)$. In particular, $y_{\varepsilon}(t):=\int_{\Omega} v_{\varepsilon}^{\chi} w_{\varepsilon}^{2}$ satisfies

$$
y_{\varepsilon}^{\prime} \leq C_{9}+C_{2} y_{\varepsilon}-C_{10} y_{\varepsilon}^{\frac{1}{a}} \forall t \in\left(0, T_{\varepsilon}\right) \text {, }
$$

which implies that $y_{\varepsilon}$ is uniformly bounded for $\varepsilon \in\left(0, \varepsilon_{p}\right)$ because $a<\frac{p}{p+1}<1$. Going back to (29), this easily yields

$$
\int_{t}^{t+1} \int_{\Omega} v_{\varepsilon}^{\chi} w_{\varepsilon x}^{2} \leq C_{11} \text { for all } t \in\left(0, T_{\varepsilon}\right) \text { and } \varepsilon \in\left(0, \varepsilon_{p}\right) \text {. }
$$


Transformed to the original variables, the latter two statements precisely establish (19) and (20).

In order to derive bounds on integrals that only involve $u_{\varepsilon}$ or $v_{\varepsilon}$ and not a combination of both functions, we next show that certain bounds for $u_{\varepsilon}$ imply similar bounds for $v_{\varepsilon}$.

Lemma 2.2

Let $\chi \in(0,1)$, and suppose that there exist $q \in[1, \infty), \tilde{\varepsilon}_{q} \in(0,1]$ and $k_{q}>0$ such that

$$
\int_{\Omega} u_{\varepsilon}^{q}(x, t) \mathrm{d} x \leq k_{q} \text { for all } t \in\left(0, T_{\varepsilon}\right) \text { and } \varepsilon \in\left(0, \tilde{\varepsilon}_{q}\right) \text {. }
$$

Then one can find $c_{q}>0$ fulfilling

$$
\int_{\Omega} v_{\varepsilon}^{q}(x, t) \mathrm{d} x \leq c_{q} \text { for all } t \in\left(0, T_{\varepsilon}\right) \text { and } \varepsilon \in\left(0, \tilde{\varepsilon}_{q}\right)
$$

Proof

We multiply the second equation in ( 8 ) by $v_{\varepsilon}^{q-1}$ and integrate over $\Omega$ to obtain

$$
\frac{1}{q} \frac{\mathrm{d}}{\mathrm{d} t} \int_{\Omega} v_{\varepsilon}^{q}=-(q-1) \varepsilon \int_{\Omega} v_{\varepsilon}^{q-2} v_{\varepsilon X}^{2}+\int_{\Omega} v_{\varepsilon}^{q-1}-\int_{\Omega} v_{\varepsilon}^{q}+\int_{\Omega} u_{\varepsilon} v_{\varepsilon}^{q-1} \text { for } t \in\left(0, T_{\varepsilon}\right)
$$

By Young's inequality, we can pick $\tilde{c}_{1}>0$ such that

$$
\int_{\Omega} v_{\varepsilon}^{q-1} \leq \frac{1}{4} \int_{\Omega} v_{\varepsilon}^{q}+\tilde{c}_{1} \text { for all } t \in\left(0, T_{\varepsilon}\right) \text { and } \varepsilon \in\left(0, \tilde{\varepsilon}_{q}\right)
$$

and

$$
\int_{\Omega} u_{\varepsilon} v_{\varepsilon}^{q-1} \leq \frac{1}{4} \int_{\Omega} v_{\varepsilon}^{q}+\tilde{c}_{1} \int_{\Omega} u_{\varepsilon}^{q} \text { for all } t \in\left(0, T_{\varepsilon}\right) \text { and } \varepsilon \in\left(0, \tilde{\varepsilon}_{q}\right) \text {. }
$$

In view of (30), (32) therefore shows that $y_{\varepsilon}(t):=\int_{\Omega} v_{\varepsilon}^{q}(x, t) \mathrm{d} x, t \in\left(0, T_{\varepsilon}\right)$, satisfies

$$
\frac{1}{q} y_{\varepsilon}^{\prime} \leq-\frac{1}{2} y_{\varepsilon}+\tilde{c}_{1}\left(1+k_{q}\right) \text { for all } t \in\left(0, T_{\varepsilon}\right) \text { and } \varepsilon \in\left(0, \tilde{\varepsilon}_{q}\right),
$$

which upon an ODE comparison argument immediately yields (31).

Now, we are in the position to prove uniform bounds, independent of both $\varepsilon$ and $t$, for the $L^{q}$ norms of $u_{\varepsilon}$ and $v_{\varepsilon}$ for any $q \in[1, \infty)$. Lemma 2.3

Let $\chi \in(0,1)$. Then for all $q \in[1, \infty)$ there exist $k_{q}>0, c_{q}>0$ and $\tilde{\varepsilon}_{q} \in\left(0, \varepsilon_{q}\right]$ such that

$$
\int_{\Omega} u_{\varepsilon}^{q}(x, t) \mathrm{d} x \leq k_{q} \text { for all } t \in\left(0, T_{\varepsilon}\right) \text { and } \varepsilon \in\left(0, \tilde{\varepsilon}_{q}\right)
$$

and

$$
\int_{\Omega} v_{\varepsilon}^{q}(x, t) \mathrm{d} x \leq c_{q} \text { for all } t \in\left(0, T_{\varepsilon}\right) \text { and } \varepsilon \in\left(0, \tilde{\varepsilon}_{q}\right) \text {. }
$$

Proof

Because $\chi<1$, it is possible to fix a number $\kappa>1$ fulfilling $\kappa<\frac{1}{\chi}$, so that $q_{k}:=\kappa^{k}, k \geq 0$, satisfies $q_{k} \rightarrow+\infty$ as $k \rightarrow \infty$. In order to prove the lemma, it is thus sufficient to show that for each fixed $k \geq 0$, we can find $A_{k}>0, B_{k}>0$, and $\tilde{\varepsilon}_{q_{k}} \in(0,1]$ such that

$$
\int_{\Omega} u_{\varepsilon}^{q_{k}}(x, t) \mathrm{d} x \leq A_{k} \text { for all } t \in\left(0, T_{\varepsilon}\right) \text { and } \varepsilon \in\left(0, \tilde{\varepsilon}_{q_{k}}\right)
$$

and

$$
\int_{\Omega} v_{\varepsilon}^{q_{k}}(x, t) \mathrm{d} x \leq B_{k} \text { for all } t \in\left(0, T_{\varepsilon}\right) \text { and } \varepsilon \in\left(0, \tilde{\varepsilon}_{q_{k}}\right)
$$

To this end, we first observe that by (12) and (13), we know that (35) and (36) hold for $k=0, \tilde{\varepsilon}_{q_{0}}:=1$ and some sufficiently large $A_{0}$ and $B_{0}$

Next, assuming the validity of (35) and (36) for some $k \geq 0, \tilde{\varepsilon}_{q_{k}} \in(0,1]$ and certain positive $A_{k}$ and $B_{k}$, we let

$$
p:=\frac{\left(q_{k}-\chi\right) \cdot q_{k+1}}{q_{k}-\chi q_{k+1}} \equiv \frac{\left(\kappa^{k}-\chi\right) \kappa}{1-\chi \kappa}
$$


where we note that the inequalities $\chi<1<\kappa<\frac{1}{\chi}$ ensure that both $\kappa^{k}-\chi$ and $1-\chi \kappa$ are positive, and that, moreover, $p>q_{k+1}>1$. Applying Lemma 2.1 to this value of $p$, in view of Hölder's inequality, we find

$$
\begin{aligned}
\int_{\Omega} u_{\varepsilon}^{q_{k+1}} & =\int_{\Omega}\left(v_{\varepsilon}^{-\frac{(p-1) \chi q_{k+1}}{p}} u_{\varepsilon}^{q_{k+1}}\right) \cdot v_{\varepsilon}^{\frac{(p-1) \chi q_{k+1}}{p}} \\
& \leq\left(\int_{\Omega} v_{\varepsilon}^{-(p-1) \chi} u_{\varepsilon}^{p}\right)^{\frac{q_{k+1}}{p}} \cdot\left(\int_{\Omega} v_{\varepsilon}^{\frac{(p-1) \chi q_{k}+1}{p-q_{k+1}}}\right)^{\frac{p-q_{k+1}}{p}} \\
& \leq C_{1} \cdot\left(\int_{\Omega} v_{\varepsilon}^{\frac{(p-1) \chi q_{k+1}}{p-q_{k+1}}}\right)^{\frac{p-q_{k+1}}{p}} \text { for all } t \in\left(0, T_{\varepsilon}\right) \text { and } \varepsilon \in\left(0, \varepsilon_{p}\right)
\end{aligned}
$$

with some $C_{1}>0$ depending on $k$ only. Because (37) entails that

$$
\frac{(p-1) \chi q_{k+1}}{p-q_{k+1}}=\frac{\frac{q_{k} q_{k+1}-q_{k}}{q_{k}-\chi q_{k}+1} \times q_{k+1}}{\frac{\chi q_{k+1}^{2}-\chi q_{k+1}}{q_{k}-\chi q_{k+1}}}=\frac{\left(q_{k+1}-1\right) q_{k} \cdot \chi q_{k+1}}{\left(q_{k+1}-1\right) \cdot \chi q_{k+1}}=q_{k},
$$

using (36) we conclude from (38) that there exists $A_{k+1}>0$ such that

$$
\int_{\Omega} u_{\varepsilon}^{q_{k+1}}(x, t) \mathrm{d} x \leq A_{k+1} \text { for all } t \in\left(0, T_{\varepsilon}\right) \text { and } \varepsilon \in\left(0, \tilde{\varepsilon}_{q_{k+1}}\right),
$$

where $\tilde{\varepsilon}_{q_{k+1}}:=\min \left\{\tilde{\varepsilon}_{q_{k}}, \varepsilon_{p}, \varepsilon_{q_{k+1}}\right\}$.

In view of Lemma 2.2, this implies that also

$$
\int_{\Omega} v_{\varepsilon}^{q_{k+1}}(x, t) \mathrm{d} x \leq B_{k+1} \text { for all } t \in\left(0, T_{\varepsilon}\right) \text { and } \varepsilon \in\left(0, \tilde{\varepsilon}_{q_{k+1}}\right)
$$

with some sufficiently large $B_{k+1}>0$. This completes the proof.

Moreover, we provide bounds on the spatial derivatives of $u_{\varepsilon}$ and $v_{\varepsilon}$. We note that unlike in the previous statements, the estimates given in the following lemma are not independent of time.

Lemma 2.4

Let $\chi \in(0,1)$. Then for all $s \in(1,2)$ and each $T \in(0, \infty)$ such that $T \leq T_{\varepsilon}$ there exists $C(s, T)>0$ such that

$$
\int_{0}^{T} \int_{\Omega}\left|u_{\varepsilon} x\right|^{s} \leq C(s, T) \text { for all } \varepsilon \in\left(0, \min \left\{\varepsilon_{2}, \tilde{\varepsilon}_{\frac{s x}{2-s}}\right\}\right)
$$

and

$$
\int_{\Omega}\left|v_{\varepsilon x}(x, t)\right|^{s} \mathrm{~d} x \leq C(s, T) \text { for all } t \in(0, T) \text { and } \varepsilon \in\left(0, \min \left\{\varepsilon_{2}, \tilde{\varepsilon}_{\frac{s x}{2-s}}\right\}\right)
$$

Proof

Writing $z_{\varepsilon}:=\frac{u_{\varepsilon}}{v_{\varepsilon}^{X}}$, from Lemma 2.1 we obtain $C_{1}=C_{1}(T)>0$ such that

$$
\int_{0}^{T} \int_{\Omega} v_{\varepsilon}^{\chi} z_{\varepsilon x}^{2} \leq C_{1} \text { for all } \varepsilon \in\left(0, \varepsilon_{2}\right)
$$

Because $v_{\varepsilon t}=\varepsilon v_{\varepsilon x x}+1-v_{\varepsilon}+u_{\varepsilon}=\varepsilon v_{\varepsilon x x}+1-v_{\varepsilon}+v_{\varepsilon}^{\chi} z_{\varepsilon}$, upon differentiation we see that

$$
v_{\varepsilon x t}=\varepsilon v_{\varepsilon x x x}-v_{\varepsilon x}+v_{\varepsilon}^{\chi} z_{\varepsilon x}+\chi v_{\varepsilon}^{\chi-1} z_{\varepsilon} v_{\varepsilon x} \text { for } x \in \Omega \text { and } t \in\left(0, T_{\varepsilon}\right) \text {. }
$$

We now fix $s \in(1,2)$, multiply both sides of this identity by $\left|v_{\varepsilon x}\right|^{s-2} v_{\varepsilon x}$ and integrate by parts to find

$$
\begin{aligned}
\frac{1}{s} \frac{\mathrm{d}}{\mathrm{d} t} \int_{\Omega}\left|v_{\varepsilon x}\right|^{s}+(s-1) \varepsilon \int_{\Omega}\left|v_{\varepsilon x}\right|^{s-2} v_{\varepsilon X X}^{2}= & -\int_{\Omega}\left|v_{\varepsilon x}\right|^{s}+\int_{\Omega} v_{\varepsilon}^{\chi}\left|v_{\varepsilon x}\right|^{s-2} v_{\varepsilon X} z_{\varepsilon X} \\
& +\chi \int_{\Omega} v_{\varepsilon}^{\chi-1} z_{\varepsilon}\left|v_{\varepsilon x}\right|^{s}
\end{aligned}
$$

for $t \in\left(0, T_{\varepsilon}\right)$ and $\varepsilon \in\left(0, \varepsilon_{2}\right)$. The second term on the right can be estimated using Young's inequality according to

$$
\int_{\Omega} v_{\varepsilon}^{\chi}\left|v_{\varepsilon x}\right|^{s-2} v_{\varepsilon X} z_{\varepsilon x} \leq \int_{\Omega} v_{\varepsilon}^{\chi} z_{\varepsilon X}^{2}+\frac{1}{4} \int_{\Omega} v_{\varepsilon}^{\chi}\left|v_{\varepsilon x}\right|^{2 s-2} \text { for all } t \in\left(0, T_{\varepsilon}\right) \text { and } \varepsilon \in\left(0, \varepsilon_{2}\right) \text {. }
$$


Because $s<2$, again by the Young inequality and Lemma 2.3, we have

$$
\begin{aligned}
\frac{1}{4} \int_{\Omega} v_{\varepsilon}^{\chi}\left|v_{\varepsilon X}\right|^{2 s-2} & \leq \int_{\Omega}\left|v_{\varepsilon X}\right|^{s}+C_{2} \int_{\Omega} v_{\varepsilon}^{\frac{s \chi}{2-s}} \\
& \leq \int_{\Omega}\left|v_{\varepsilon X}\right|^{s}+C_{3} \text { for all } t \in\left(0, T_{\varepsilon}\right) \text { and } \varepsilon \in\left(0, \tilde{\varepsilon}_{\frac{s \chi}{2-s}}\right)
\end{aligned}
$$

with some $C_{2}>0$ and $C_{3}>0$ depending on $s$ only. As to the third term on the right of (42), recalling (11) and that $\chi<1$, we obtain $C_{4}>0$ such that

$$
\chi \int_{\Omega} v_{\varepsilon}^{\chi-1} z_{\varepsilon}\left|v_{\varepsilon X}\right|^{s} \leq C_{4}\left\|Z_{\varepsilon}(\cdot, t)\right\|_{L^{\infty}(\Omega)} \cdot \int_{\Omega}\left|v_{\varepsilon x}\right|^{s} \text { for all } t \in\left(0, T_{\varepsilon}\right) \text { and } \varepsilon \in\left(0, \varepsilon_{2}\right)
$$

Because (41) in conjunction with (11) and Lemma 2.1 implies that

$$
\int_{0}^{T}\left\|z_{\varepsilon}(\cdot, t)\right\|_{W^{1,2}(\Omega)}^{2} d t \leq C_{5} \text { for all } \varepsilon \in\left(0, \varepsilon_{2}\right)
$$

with some $C_{5}=C_{5}(T)>0$, and because $W^{1,2}(\Omega) \hookrightarrow L^{\infty}(\Omega)$, we infer from (42)-(45) that

$$
\frac{1}{s} \frac{\mathrm{d}}{\mathrm{d} t} \int_{\Omega}\left|v_{\varepsilon x}\right|^{s} \leq f_{\varepsilon}(t) \cdot \int_{\Omega}\left|v_{\varepsilon x}\right|^{s}+g_{\varepsilon}(t) \text { for all } t \in(0, T)
$$

where $f_{\varepsilon}(t):=C_{4}\left\|z_{\varepsilon}(\cdot, t)\right\|_{L}(\Omega)$ and $g_{\varepsilon}(t):=\int_{\Omega} v_{\varepsilon}^{X} z_{\varepsilon X}^{2}+C_{3}$ satisfy

$$
\int_{0}^{T}\left|f_{\varepsilon}(t)\right| \mathrm{d} t \leq \sqrt{T} \cdot\left(\int_{0}^{T} f_{\varepsilon}^{2}(t) \mathrm{d} t\right)^{\frac{1}{2}} \leq \sqrt{2 C_{5} T} C_{4}
$$

and

$$
\int_{0}^{T}\left|g_{\varepsilon}(t)\right| \mathrm{d} t \leq C_{1}+C_{3} T
$$

Consequently, since an integration of (46) yields

$$
\int_{\Omega}\left|v_{\varepsilon x}(x, t)\right|^{s} \mathrm{~d} x \leq e^{s \cdot \int_{0}^{t}\left|f_{\varepsilon}(\tau)\right| \mathrm{d} \tau} \cdot \int_{\Omega}\left|v_{0 x}(x)\right|^{s} \mathrm{~d} x+\int_{0}^{t} e^{s \cdot \int_{\tau}^{t}\left|f_{\varepsilon}(\sigma)\right| \mathrm{d} \sigma} \cdot\left|g_{\varepsilon}(\tau)\right| \mathrm{d} \tau
$$

for all $t \in(0, T)$ and $\varepsilon \in\left(0, \min \left\{\varepsilon_{2}, \tilde{\varepsilon}_{\frac{s \chi}{2-s}}\right\}\right)$, the inequality (40) results from (47) and (48) for some suitably large $C(s, T)$. Once more using that $u_{\varepsilon x}=\chi v_{\varepsilon}^{\chi-1} Z_{\varepsilon} v_{\varepsilon X}+v_{\varepsilon}^{\chi} Z_{\varepsilon X}$, we now only need to combine (40) with (41), Lemma 2.3 and (11) to easily conclude that (39) holds if we adequately enlarge $C(s, T)$.

Next, we use the estimates obtained so far to show that if $\varepsilon$ is small enough then $\left(u_{\varepsilon}, v_{\varepsilon}\right)$ exists globally in time and enjoys some $\varepsilon$-independent local-in-time boundedness property.

Corollary 2.5

Suppose that $\chi \in(0,1)$. Then there is $\tilde{\varepsilon} \in(0,1]$ such that for any $\varepsilon \in(0, \tilde{\varepsilon})$ the solution $\left(u_{\varepsilon}, v_{\varepsilon}\right)$ of $(8)$ is global in time. Moreover, for any $T>0$ there is $\tilde{C}_{T}>0$ such that

$$
\left\|u_{\varepsilon}\right\|_{L^{\infty}(\Omega \times(0, T))}+\left\|v_{\varepsilon}\right\|_{L^{\infty}(\Omega \times(0, T))} \leq \tilde{C}_{T} \text { for all } \varepsilon \in(0, \tilde{\varepsilon})
$$

Proof

We fix $q \in(1,2)$, set $r:=\frac{q+1}{2} \in(1, q)$, define $\tilde{\varepsilon}:=\min \left\{\varepsilon_{2}, \tilde{\varepsilon}_{\frac{q \chi}{2-q}}, \tilde{\varepsilon}_{r}, \tilde{\varepsilon}_{\frac{q r}{q-r}}\right\} \in(0,1]$ and fix $T \in(0, \infty)$ such that $T \leq T_{\varepsilon}$. Then by Lemma 2.3 and Lemma 2.4 , we deduce that there is $C_{1}=C_{1}(T)>0$ such that

$$
\left\|v_{\varepsilon}(\cdot, t)\right\|_{W^{1, q}(\Omega)} \leq C_{1} \text { for all } t \in(0, T)
$$


Next, we define the operator $A:=-\Delta$ with $D(A):=\left\{f \in W^{2, r}(\Omega) \mid f_{x}=0\right.$ on $\left.\partial \Omega\right\}$ and fix $\beta \in\left(\frac{1}{2 r}, \frac{1}{2}\right)$ and $\delta \in\left(0, \frac{1}{2}-\beta\right)$. Because $D\left((A+1)^{\beta}\right) \hookrightarrow C^{0}(\bar{\Omega})$, we conclude using [18, Section 2], (11), Hölder's inequality, Lemma 2.3, (49) and the notations from the proof of Lemma 1.2 that

$$
\begin{aligned}
\left\|u_{\varepsilon}(, t)\right\|_{C^{0}(\bar{\Omega})}= & \left\|\varphi+e^{t \Delta} \tilde{u}_{0}+\int_{0}^{t} e^{(t-s) \Delta}\left[-\nabla \cdot\left(\chi \frac{\tilde{u}_{\varepsilon}+\varphi}{\rho\left(v_{\varepsilon}\right)} \nabla v_{\varepsilon}\right)-\lambda\left(\tilde{u}_{\varepsilon}+\varphi\right)+\varphi_{X X}\right] \mathrm{d} s\right\|_{C^{0}(\bar{\Omega})} \\
\leq & \|\varphi\|_{C^{0}(\bar{\Omega})}+\left\|e^{t \Delta}\left(u_{0}-\varphi\right)\right\|_{C^{0}(\bar{\Omega})} \\
& +C_{2} \int_{0}^{t}\left\|(A+1)^{\beta} e^{-(t-s) A} \nabla \cdot\left(\chi \frac{u_{\varepsilon}}{v_{\varepsilon}} \nabla v_{\varepsilon}\right)\right\|_{L^{r}(\Omega)} \mathrm{d} s \\
& +C_{3} \int_{0}^{t}\left\|(A+1)^{\beta} e^{-(t-s) A}\left(\lambda u_{\varepsilon}+\varphi_{X X}\right)\right\|_{L^{r}(\Omega)} \mathrm{d} s \\
\leq & C_{4}\left(\|\varphi\|_{C^{0}(\bar{\Omega})}+\left\|u_{0}\right\|_{C^{0}(\bar{\Omega})}\right)+C_{5} \int_{0}^{t}(t-s)^{-\beta-\frac{1}{2}-\delta}\left\|\chi \frac{u_{\varepsilon}}{v_{\varepsilon}} \nabla v_{\varepsilon}\right\|_{L^{r}(\Omega)} \mathrm{d} s \\
& +C_{6} \int_{0}^{t}(t-s)^{-\beta}\left\|\lambda u_{\varepsilon}+\varphi_{X x}\right\|_{L^{r}(\Omega)} \mathrm{d} s \\
\leq & C_{4}\left(\|\varphi\|_{C^{0}(\bar{\Omega})}+\left\|u_{0}\right\|_{C^{0}(\bar{\Omega})}\right) \\
& +C_{5} \frac{\chi}{C_{0}} \int_{0}^{t}(t-s)^{-\beta-\frac{1}{2}-\delta}\left\|u_{\varepsilon}\right\|_{L^{\frac{r q}{q-r}}(\Omega)}\left\|\nabla v_{\varepsilon}\right\|_{L^{q}(\Omega)} \mathrm{d} s \\
& +C_{6} \int_{0}^{t}(t-s)^{-\beta}\left(\lambda\left\|u_{\varepsilon}\right\|_{L^{r}(\Omega)}+\left\|\varphi_{x x}\right\|_{L^{r}(\Omega)}\right) \mathrm{d} s \\
\leq & C_{4}\left(\|\varphi\|_{C^{0}(\bar{\Omega})}+\left\|u_{0}\right\|_{C^{0}(\bar{\Omega})}\right)+C_{7} \frac{\chi}{C_{0}}\left(k_{\frac{1 q}{q-r}}\right)^{\frac{q-r}{q r} C_{1} T^{\frac{1}{2}-\beta-\delta}} \\
& +C_{8}\left(k_{r}^{\frac{1}{r}}+\left\|\varphi_{x x}\right\|_{L^{r}(\Omega)}\right) T^{1-\beta}, \text { for all } t \in(0, T) .
\end{aligned}
$$

Combining this estimate with (49), we deduce that $\left(u_{\varepsilon}, v_{\varepsilon}\right)$ exists globally in time because otherwise the choice $T=T_{\varepsilon}<\infty$ would contradict (9). Hence, the claim follows from both estimates as $W^{1,9}(\Omega) \hookrightarrow L^{\infty}(\Omega)$.

Now, we are in the position to prove that $\left(u_{\varepsilon}, v_{\varepsilon}\right)$ converge to a global weak solution $(u, v)$ of $(6)$ as $\varepsilon \searrow 0$ along a suitable subsequence.

Lemma 2.6

Let $\chi \in(0,1)$. Then there exists a sequence of numbers $\varepsilon=\varepsilon_{j} \searrow 0$ along that we have

$$
\begin{gathered}
u_{\varepsilon} \stackrel{\star}{\rightarrow} u \text { in } L_{\mathrm{loc}}^{\infty}(\bar{\Omega} \times[0, \infty)), \\
u_{\varepsilon} \rightarrow u \text { in } L_{\mathrm{loc}}^{s}\left([0, \infty) ; W^{1, s}(\Omega)\right), \\
v_{\varepsilon} \stackrel{\star}{\rightarrow} v \text { in } L_{\mathrm{loc}}^{\infty}\left([0, \infty) ; W^{1, s}(\Omega)\right), \\
u_{\varepsilon} \rightarrow u \text { in } L_{\mathrm{loc}}^{s}\left([0, \infty) ; L^{p}(\Omega)\right) \text { and a.e. in } \Omega \times(0, \infty), \\
v_{\varepsilon} \rightarrow v \operatorname{in} L_{\mathrm{loc}}^{p}\left([0, \infty) ; L^{p}(\Omega)\right) \text { and a.e. in } \Omega \times(0, \infty),
\end{gathered}
$$

for all $s \in(1,2)$ and all $p \in(1, \infty)$ and some pair $(u, v)$ of non-negative functions satisfying

$$
u \in L_{\mathrm{loc}}^{\infty}(\bar{\Omega} \times[0, \infty)) \cap \bigcap_{s \in(1,2)} L_{\mathrm{loc}}^{s}\left([0, \infty) ; W^{1, s}(\Omega)\right)
$$

and

$$
v \in L_{\mathrm{loc}}^{\infty}(\bar{\Omega} \times[0, \infty)) \cap \bigcap_{s \in(1,2)} L_{\text {loc }}^{\infty}\left([0, \infty) ; W^{1, s}(\Omega)\right) .
$$

Moreover, $(u, v)$ is a global weak solution of $(6)$ in the sense of Definition 1.1. 
Proof

We fix $T>0, p \in(1, \infty)$ and $s \in(1,2)$, set $s^{\prime}:=\frac{s}{s-1}$ and choose $q \in(1, \infty)$ such that $\frac{1}{q}<\frac{1}{s}-\frac{1}{2}$. Moreover, we define $\tilde{s}:=\left(\frac{1}{s}-\frac{1}{q}\right)^{-1} \in(s, 2)$ and $\bar{\varepsilon}:=\min \left\{\tilde{\varepsilon}_{,} \tilde{\varepsilon}_{2}, \tilde{\varepsilon}_{q}, \varepsilon \frac{s \chi}{2-s}, \varepsilon \frac{\tilde{s} \chi}{2-\tilde{s}}\right\}$.

We now show that $u_{\varepsilon t}$ is uniformly bounded in $L^{s}\left((0, T) ; W^{-1, s^{\prime}}(\Omega)\right)$ for $\varepsilon \in(0, \bar{\varepsilon})$. For $\psi \in L^{s^{\prime}}\left((0, T) ; W^{1, s^{\prime}}(\Omega)\right)$ we obtain

$$
<u_{\varepsilon t}, \psi>_{W-1, s^{\prime}(\Omega), W^{1, s^{\prime}}(\Omega)}=-\int_{\Omega} u_{\varepsilon x} \psi_{X}+\chi \int_{\Omega} \frac{u_{\varepsilon}}{v_{\varepsilon}} v_{\varepsilon X} \psi_{X}-\lambda \int_{\Omega} u_{\varepsilon} \psi+\nu \psi(0) .
$$

Because $\frac{1}{q}+\frac{1}{\tilde{s}}+\frac{1}{s^{\prime}}=1$, by (11), Lemma 2.3 and Lemma 2.4 we have

$$
\begin{gathered}
v|\psi(0)| \leq C_{1}\|\psi(t)\|_{W^{1, s^{\prime}}(\Omega)^{\prime}} \\
\int_{\Omega}\left|u_{\varepsilon x} \psi_{X}+\lambda u_{\varepsilon} \psi\right|(t) \leq C\left\|u_{\varepsilon}(t)\right\|_{W^{1, s}(\Omega)}\|\psi(t)\|_{W^{1, s^{\prime}}(\Omega)} \leq C_{2}(t)\|\psi(t)\|_{W^{1, s^{\prime}}(\Omega)^{\prime}} \\
\chi \int_{\Omega}\left|\frac{u_{\varepsilon}}{v_{\varepsilon}} v_{\varepsilon X} \psi_{X}\right|(t) \leq \frac{\chi}{C_{0}}\left\|u_{\varepsilon}(t)\right\|_{L^{q}(\Omega)}\left\|v_{\varepsilon X}(t)\right\|_{L^{s}}\|\psi(t)\|_{W^{1, s^{\prime}}(\Omega)} \leq C_{3}(t)\|\psi(t)\|_{W^{1, s^{\prime}}(\Omega)}
\end{gathered}
$$

with $C_{2} \in L^{s}((0, T)), C_{3} \in L^{\infty}((0, T))$. This implies

$$
\left\|u_{\varepsilon t}(t)\right\|_{W^{-1, s^{\prime}}(\Omega)} \leq C_{1}+C_{2}(t)+C_{3}(t) \text { for any } \varepsilon \in(0, \bar{\varepsilon}),
$$

and, therefore, due to Lemma 2.3 and Lemma 2.4 ,

$$
\left\|u_{\varepsilon t}\right\|_{L^{s}\left((0, T) ; W^{1,-s^{\prime}}(\Omega)\right)}+\left\|u_{\varepsilon}\right\|_{L^{s}\left((0, T) ; W^{1, s}(\Omega)\right)} \leq k \text { for any } \varepsilon \in(0, \bar{\varepsilon}),
$$

where $k$ is independent of $\varepsilon \in(0, \bar{\varepsilon})$. Because $W^{1, s}(\Omega) \hookrightarrow L^{p}(\Omega)$ is a compact inclusion and $L^{p}(\Omega) \hookrightarrow W^{-1, s^{\prime}}(\Omega)$, by $(55)$ and the Aubin-Lions Theorem, we conclude that

$$
u_{\varepsilon} \rightarrow u \text { in } L^{s}\left((0, T) ; L^{p}(\Omega)\right)
$$

along a suitable subsequence. This yields (53) and hence (50) and (51) follow from Corollary 2.5, Lemma 2.3, and Lemma 2.4.

Similarly, we obtain

$$
\begin{aligned}
& \mid\left\langle v_{\varepsilon t}(t), \psi(t)>_{W^{-1, s^{\prime}}(\Omega), W^{1, s^{\prime}}(\Omega) \mid} \leq \varepsilon \int_{\Omega}\left|v_{\varepsilon x} \psi_{x}\right|(t)+\int_{\Omega}\left|1-v_{\varepsilon}+u_{\varepsilon} \| \psi\right|(t)\right. \\
& \leq c\left(1+\left\|v_{\varepsilon}(t)\right\|_{W^{1, s}(\Omega)}+\left\|u_{\varepsilon}(t)\right\|_{L^{s}(\Omega)}\right)\|\psi(t)\|_{W^{1, s^{\prime}}(\Omega)} .
\end{aligned}
$$

Hence, Lemma 2.3 and Lemma 2.4 imply

$$
\left\|v_{\varepsilon t}\right\|_{L^{p}\left((0, T) ; W^{1,-s^{\prime}}(\Omega)\right)}+\left\|v_{\varepsilon}\right\|_{L^{p}\left((0, T) ; W^{1, s}(\Omega)\right)} \leq \tilde{k} \text { for any } \varepsilon \in(0, \bar{\varepsilon})
$$

and again by the Aubin-Lions Theorem, we deduce that

$$
v_{\varepsilon} \rightarrow v \text { in } L^{p}\left((0, T) ; L^{p}(\Omega)\right)
$$

along a suitable subsequence. In conjunction with Lemma 2.3 and Lemma 2.4 this proves (54) and (52).

Next, we verify that $(u, v)$ is a global weak solution of $(6)$. Because the mean value theorem and (11) imply

$$
\left|\frac{1}{v_{\varepsilon}}-\frac{1}{v}\right| \leq \frac{1}{c_{0}^{2}}\left|v_{\varepsilon}-v\right|,
$$

we obtain

$$
\frac{1}{V_{\varepsilon}} \rightarrow \frac{1}{V} L^{p}(\Omega \times(0, T)) \text { for any } p \in(1, \infty)
$$

by (57). Thus, due to (52), (56), and (58) we deduce that

$$
\chi \int_{0}^{\infty} \int_{\Omega} \frac{u_{\varepsilon}}{v_{\varepsilon}} v_{\varepsilon x} \varphi_{X} \rightarrow \chi \int_{0}^{\infty} \int_{\Omega} \frac{u}{v} v_{X} \varphi_{X} \text { as } \varepsilon \rightarrow 0
$$

for all $\varphi \in C_{0}^{\infty}(\bar{\Omega} \times[0, \infty))$. As the limit $\varepsilon \rightarrow 0$ in all other terms in the weak formulation of (8) can be taken in a standard way due to (50)-(54), we conclude that $(u, v)$ is a weak solution of $(6)$ in the sense of Definition 1.1. 
Using an $L^{p}$ iteration of Moser-type, we are now able to prove that $u$ and $v$ are in fact uniformly bounded in $\Omega \times(0, \infty)$.

Lemma 2.7

Let $\chi \in(0,1)$. Then there exist $k_{\infty}>0$ and $c_{\infty}>0$ such that

$$
\|u\|_{L}(\Omega \times(0, \infty)) \leq k_{\infty} \text { and }\|v\|_{L}(\Omega \times(0, \infty)) \leq c_{\infty}
$$

Proof

We fix $p \geq 2, \varepsilon \in\left(0, \min \left\{\varepsilon_{p}, \tilde{\varepsilon}\right\}\right)$, set $z_{\varepsilon}:=\frac{u_{\varepsilon}}{v_{\varepsilon}^{\chi}}$ and conclude by (24) that

$$
\frac{\partial}{\partial t} \int_{\Omega} v_{\varepsilon}^{\chi} z_{\varepsilon}^{p}+A_{p} \int_{\Omega} v_{\varepsilon}^{\chi}\left|\left(z_{\varepsilon}^{\frac{p}{2}}\right)_{X}\right|^{2} \leq B_{p} \int_{\Omega} v_{\varepsilon}^{\chi} z_{\varepsilon}^{p}+1
$$

with

$$
A_{p}:=\frac{p-1}{p}, \text { and } B_{p}:=\max \left\{(p-1) v^{\frac{p}{p-1}}\left(1+\frac{p v^{\frac{p}{p-1}}}{2 c_{0}^{\chi}}\right) c_{0}^{-\chi}+(p-1) \chi-p \lambda, 0\right\}
$$

We next apply Hölder's inequality and (13) to obtain

$$
\int_{\Omega} v_{\varepsilon}^{\chi} Z_{\varepsilon}^{p} \leq\left(\int_{\Omega} v_{\varepsilon}\right)^{\chi}\left(\int_{\Omega} z_{\varepsilon}^{\frac{p}{1-\chi}}\right)^{1-\chi} \leq c_{1}^{\chi}\left(\int_{\Omega} z_{\varepsilon}^{\frac{p}{1-\chi}}\right)^{1-\chi}
$$

Observe that the Gagliardo-Nirenberg inequality implies

$$
\left(\int_{\Omega} z_{\varepsilon}^{\frac{p}{1-\chi}}\right)^{1-\chi}=\left\|z_{\varepsilon}^{\frac{p}{2}}\right\|_{L^{1-\chi}(\Omega)}^{2} \leq C_{1}\left(\left\|z_{\varepsilon}^{\frac{p}{2}}\right\|_{L^{1}(\Omega)}^{2(1-a)}\left\|\left(z_{\varepsilon}^{\frac{p}{2}}\right)_{X}\right\|_{L^{2}(\Omega)}^{2 a}+\left\|z_{\varepsilon}^{\frac{p}{2}}\right\|_{L^{1}(\Omega)}^{2}\right),
$$

where $a=\frac{1+\chi}{3} \in\left(\frac{1}{3}, \frac{2}{3}\right)$ and $C_{1}=C_{1}(\Omega, \chi)$ is a positive constant. Hence, for any $\delta>0$, we deduce by (61), (62), Young's inequality, and (11) that

$$
\begin{aligned}
\int_{\Omega} v_{\varepsilon}^{\chi} Z_{\varepsilon}^{p} & \leq\left(\frac{\left(C_{1} c_{1}^{\chi}\right)^{\frac{1}{1-a}}(1-a) a^{\frac{a}{1-a}}}{\left(\delta c_{0}^{\chi}\right)^{\frac{a}{1-a}}}+C_{1} c_{1}^{\chi}\right)\left\|z_{\varepsilon}^{\frac{p}{2}}\right\|_{L^{1}(\Omega)}^{2}+\delta c_{0}^{\chi}\left\|\left(z_{\varepsilon}^{\frac{p}{2}}\right)_{X}\right\|_{L^{2}(\Omega)}^{2} \\
& \leq\left(\frac{\left(C_{1} c_{1}^{\chi}\right)^{\frac{1}{1-a}(1-a) a^{\frac{a}{1-a}}}}{\left(\delta c_{0}^{\chi}\right)^{\frac{a}{1-a}}}+C_{1} c_{1}^{\chi}\right) c_{0}^{-2 \chi}\left\|v_{\varepsilon}^{\chi} Z_{\varepsilon}^{\frac{p}{2}}\right\|_{L^{1}(\Omega)}^{2}+\delta\left\|v_{\varepsilon}^{\frac{\chi}{2}}\left(z_{\varepsilon}^{\frac{p}{2}}\right)_{X}\right\|_{L^{2}(\Omega)^{2}}^{2}
\end{aligned}
$$

Setting now

$$
\delta:=\frac{A_{p}}{2\left(1+B_{p}\right)}
$$

by $(60)$ and (63) we conclude that

$$
\frac{\partial}{\partial t} \int_{\Omega} v_{\varepsilon}^{\chi} z_{\varepsilon}^{p}+\int_{\Omega} v_{\varepsilon}^{\chi} Z_{\varepsilon}^{p} \leq D_{p}\left(\int_{\Omega} v_{\varepsilon}^{\chi} z_{\varepsilon}^{\frac{p}{2}}\right)^{2}+1
$$

where

$$
D_{p}:=\left(1+B_{p}\right)\left(\frac{\left(C_{1} C_{1}^{\chi}\right)^{\frac{1}{1-a}}(1-a) a^{\frac{a}{1-a}}}{\left(\delta c_{0}^{\chi}\right)^{\frac{a}{1-a}}}+C_{1} c_{1}^{\chi}\right) c_{0}^{-2 \chi} \leq c p^{2 \frac{3}{2-\chi}} \leq c p^{6},
$$

and $c \geq 1$ is independent of $p \geq 2$ and depends on $\chi \in(0,1), \Omega$, $\nu$ and $\lambda$. Now, (64) implies

$$
\int_{\Omega}\left(v_{\varepsilon}^{\chi} z_{\varepsilon}^{p}\right)(t) \leq \max \left\{c p^{6} \sup _{t \in(0, T)}\left(\int_{\Omega} v_{\varepsilon}^{\chi} z_{\varepsilon}^{\frac{p}{2}}\right)^{2}+1, \int_{\Omega} v_{0}^{\chi} z_{0}^{p}\right\}
$$

for any $t \in(0, T)$ and $\varepsilon \in\left(0, \min \left\{\varepsilon_{p}, \tilde{\varepsilon}\right\}\right)$, where $T \in(0, \infty)$ is arbitrary and $z_{0}:=\frac{u_{0}}{v_{0}^{x}}$.

Next, we pass to the limit as $\varepsilon \rightarrow 0$, where $T$ will be kept fixed. We fix $q>0$ and $r \geq 0$ and set $w_{\varepsilon}:=v_{\varepsilon}^{-r} u_{\varepsilon}^{q}$. Then by (11) and Lemma 2.3 , we conclude that

$$
\int_{\Omega} w_{\varepsilon}^{2}(t) \leq c_{0}^{-2 r} k_{2 q} \text { for all } t>0 \text { and any } \varepsilon \in\left(0, \min \left\{\tilde{\varepsilon}_{2 q}, \tilde{\varepsilon}\right\}\right)
$$


Hence, for a.e. $t \in(0, T)$ there is $w(t) \in L^{2}(\Omega)$ such that $w_{\varepsilon}(t) \rightarrow w(t)$ in $L^{2}(\Omega)$. Introducing $\Psi(x) \equiv 1, x \in \Omega$, we therefore conclude that

$$
\int_{\Omega} w_{\varepsilon}(t)=\int_{\Omega} w_{\varepsilon}(t) \Psi \rightarrow \int_{\Omega} w(t) \Psi=\int_{\Omega} w(t) \text { for a.e. } t \in(0, T) .
$$

As furthermore $w_{\varepsilon} \rightarrow v^{-r} u^{q}$ a.e. in $\Omega \times(0, T)$ by Lemma 2.6, Egorov's theorem implies $w=v^{-r} u^{q}$ a.e. in $\Omega \times(0, T)$ and

$$
\int_{\Omega}\left(v_{\varepsilon}^{-r} u_{\varepsilon}^{q}\right)(t) \rightarrow \int_{\Omega}\left(v^{-r} u^{q}\right)(t) \text { for a.e. } t \in(0, T),
$$

where $T>0, q>0$ and $r \geq 0$ are arbitrary. Thus, because $T>0$ is arbitrary, by (65) and (66) we deduce that

$$
\int_{\Omega}\left(v^{\chi} Z^{p}\right)(t) \leq \max \left\{c p^{6} \sup _{t \in(0, \infty)}\left(\int_{\Omega} v^{\chi} Z^{\frac{p}{2}}\right)^{2}+1, \int_{\Omega} v_{0}^{\chi} z_{0}^{p}\right\}
$$

for a.e. $t \in(0, \infty)$ and any $p \geq 2$, where $c \geq 1$ is independent of $p$ and $z:=\frac{u}{v^{\chi}}$.

In order to perform a Moser iteration, we set $p_{k}:=2^{k}, k \in \mathbb{N}_{0}:=\mathbb{N} \cup\{0\}$,

$$
M_{k}:=\sup _{t \in(0, \infty)} \int_{\Omega} v^{\chi} z^{2^{k}} \text { and } K:=\max \left\{k_{1},\left\|z_{0}\right\|_{L^{\infty}(\Omega)}\left(1+\left\|v_{0}\right\|_{L^{\infty}(\Omega)}^{\chi}\right), 1\right\},
$$

where $k_{1}$ is defined in (12). Hence, we obtain

$$
M_{0}=\sup _{t \in(0, \infty)} \int_{\Omega} v^{\chi} z=\sup _{t \in(0, \infty)} \int_{\Omega} u \leq k_{1} \leq K,
$$

due to (12) and (66). Moreover, we have

$$
M_{k} \leq \max \left\{c 2^{6 k} M_{k-1}^{2}+1, K^{2^{k}}\right\}, k \geq 1
$$

In order to show that $\sup _{k \in \mathbb{N}_{0}} M_{k}^{\frac{1}{2^{k}}}<\infty$, we consider $N_{k}$ defined by

$$
N_{k}=c 2^{6 k+1} N_{k-1}^{2}, k \geq 1, N_{0}=K .
$$

As $k \geq 1$ and $c \geq 1$, it is clear by induction that $M_{k} \leq N_{k}$ for $k \in \mathbb{N}_{0}$. Taking $\log _{2}$ and defining $Y_{k}:=\log _{2} N_{k}$, we deduce that

$$
Y_{k}=2 Y_{k-1}+6 k+1+\log _{2} c, k \geq 1, Y_{0}=\log _{2} K
$$

Standard computations now show that

$$
Y_{k}=2^{k}\left(\log _{2} k+\sum_{j=1}^{k} \frac{6 j+1+\log _{2} c}{2^{j}}\right) \leq 2^{k}\left(\log _{2} k+\sum_{j=1}^{\infty} \frac{6 j+1+\log _{2} c}{2^{j}}\right)=: 2^{k} S
$$

Hence, we conclude that

$$
M_{k}^{\frac{1}{2^{k}}} \leq N_{k}^{\frac{1}{2^{k}}}=2^{Y_{k} \cdot \frac{1}{2^{k}}}=2^{S}, k \in \mathbb{N}_{0}
$$

so that (11) implies

$$
\begin{aligned}
\sup _{t \in(0, \infty)}\|z\|_{L^{\infty}(\Omega)} & \leq \limsup _{k \rightarrow \infty} \sup _{t \in(0, \infty)}\|z\|_{L^{2^{k}}(\Omega)} \leq \limsup _{k \rightarrow \infty} \sup _{t \in(0, \infty)} c_{0}^{-\frac{\chi}{2^{k}}}\left|\int_{\Omega} v^{\chi} Z^{2^{k}}\right|^{\frac{1}{2^{k}}} \\
& \leq \limsup _{k \rightarrow \infty} c_{0}^{-\frac{1}{2^{k}} 2^{S}=2^{S}}
\end{aligned}
$$

Moreover, for all $\varphi \in C_{0}^{\infty}(\bar{\Omega} \times[0, \infty))$ with $\varphi \geq 0, v$ satisfies

$$
\begin{aligned}
-\int_{0}^{\infty} \int_{\Omega} v \varphi_{t}-\int_{\Omega} v_{0}(x) \varphi(x, 0) \mathrm{d} x & =\int_{0}^{\infty} \int_{\Omega}\left(1-v+v^{\chi} z\right) \varphi \leq \int_{0}^{\infty} \int_{\Omega}\left(1-v+2^{S} v \chi\right) \varphi \\
& \leq \int_{0}^{\infty} \int_{\Omega}\left(1+(1-\chi) 2^{\frac{s}{1-\chi}}-(1-\chi) v\right) \varphi
\end{aligned}
$$

due to $\chi \in(0,1)$ and Young's inequality. Thus, we conclude by a weak comparison argument that

$$
v \leq \max \left\{\left\|v_{0}\right\|_{L} \infty(\Omega), \frac{1}{1-\chi}+2^{\frac{s}{1-\chi}}\right\} \text { in } \bar{\Omega} \times[0, \infty) .
$$


Therefore,

$$
u=z v^{\chi} \leq 2^{S}\left(\max \left\{\left\|v_{0}\right\|_{L^{\infty}(\Omega)}, \frac{1}{1-\chi}+2^{\frac{s}{1-\chi}}\right\}\right)^{\chi} \text { in } \bar{\Omega} \times[0, \infty),
$$

which completes the proof.

In order to show the convergence of $(u, v)$ to the steady state of $(6)$ as $t \rightarrow \infty$, it is useful to have uniform bounds on $u$ and $v$ that are independent of the initial data. These bounds are established in the following corollary for large times if $\chi$ is bounded away from 1.

Corollary 2.8

Let $\lambda_{0}>0$ and $\chi_{0} \in(0,1)$. Then there exists $C_{\infty}=C_{\infty}\left(\lambda_{0}, \chi_{0}\right)>0$ depending only on $v, \lambda_{0}$, and $\chi_{0}$ such that for all $\lambda>\lambda_{0}$ and all $\chi \in\left(0, \chi_{0}\right)$

$$
\left\|\frac{u}{v \chi}\right\|_{L^{\infty}\left(\Omega \times\left(t_{0}, \infty\right)\right)} \leq C_{\infty}
$$

as well as

$$
v \geq \frac{1}{2} \text { a.e. in } \bar{\Omega} \times\left[t_{0}, \infty\right)
$$

where the constant $t_{0}>0$ depends on $v, \lambda_{0}, \chi_{0}, u_{0}$ and $v_{0}$.

Proof

By (16) and (17), there is $t_{1}>0$ such that

$$
v_{\varepsilon} \geq \frac{1}{2}=: \tilde{c}_{0} \quad \text { in } \bar{\Omega} \times\left[t_{1}, \infty\right) \text { and } \int_{\Omega} u_{\varepsilon}(x, t) \mathrm{d} x \leq \frac{2 \nu}{\lambda_{0}}=: \tilde{k}_{1} \quad \text { for } t \in\left[t_{1}, \infty\right) .
$$

Hence, we have

$$
\frac{\mathrm{d}}{\mathrm{d} t} \int_{\Omega} v_{\varepsilon}(x, t) \mathrm{d} x \leq 1+\tilde{k}_{1}-\int_{\Omega} v_{\varepsilon}(x, t) \mathrm{d} x, t \geq t_{1},
$$

so that the comparison principle and (13) imply

$$
\int_{\Omega} v_{\varepsilon}(x, t) \mathrm{d} x \leq\left(c_{1}-1-\tilde{k}_{1}\right) e^{-\left(t-t_{1}\right)}+1+\tilde{k}_{1}, t \in\left[t_{1}, \infty\right) .
$$

Thus, we can pick $\tilde{t}_{1} \geq t_{1}$ (for simplicity of the notation we drop the tilde) such that (69) and

$$
\int_{\Omega} v_{\varepsilon}(x, t) \mathrm{d} x \leq 2+\tilde{k}_{1}=: \tilde{c}_{1} \quad \text { for } t \in\left[t_{1}, \infty\right)
$$

are fulfilled. Observe that $\tilde{c}_{0}, \tilde{k}_{1}$, and $\tilde{c}_{1}$ only depend on $v$ and $\lambda_{0}$.

Next, we fix $p \geq 2, \varepsilon \in\left(0, \min \left\{\varepsilon_{p}, \tilde{\varepsilon}\right\}\right), \chi \in\left(0, \chi_{0}\right)$, and set $z_{\varepsilon}:=\frac{u_{\varepsilon}}{v_{\varepsilon}^{X}}$ as well as $z:=\frac{u}{v^{X}}$. Using now (69) and (70) instead of (11)-(13), we proceed similar to $(60)-(62)$ and conclude that

$$
\frac{\partial}{\partial t} \int_{\Omega} v_{\varepsilon}^{\chi} Z_{\varepsilon}^{p}+A_{p} \int_{\Omega} v_{\varepsilon}^{\chi}\left|\left(z_{\varepsilon}^{\frac{p}{2}}\right)_{x}\right|^{2} \leq \tilde{B}_{p} \int_{\Omega} v_{\varepsilon}^{\chi} Z_{\varepsilon}^{p}+1, \text { for } t \geq t_{1}
$$

where

$$
A_{p}:=\frac{p-1}{p}, \text { and } \tilde{B}_{p}:=\max \left\{(p-1) v^{\frac{p}{p-1}}\left(1+\frac{p v^{\frac{p}{p-1}}}{2 \tilde{c}_{0}^{\chi}}\right) \tilde{c}_{0}^{-\chi}+(p-1) \chi-p \lambda, 0\right\}
$$

and

$$
\begin{aligned}
\int_{\Omega} v_{\varepsilon}^{\chi} z_{\varepsilon}^{p} & \leq \tilde{C}_{1}^{\chi}\left\|z_{\varepsilon}^{\frac{p}{2}}\right\|_{L^{1-\chi}(\Omega)}^{2} \leq\left(1+\tilde{C}_{1}^{\chi 0}\right) \tilde{C}_{1}\left(\left\|z_{\varepsilon}^{\frac{p}{2}}\right\|_{L^{1}(\Omega)}^{2(1-a)}\left\|\left(z_{\varepsilon}^{\frac{p}{2}}\right)_{X}\right\|_{L^{2}(\Omega)}^{2 a}+\left\|z_{\varepsilon}^{\frac{p}{2}}\right\|_{L^{1}(\Omega)}^{2}\right) \\
& \leq 2\left[\left(\left(1+\tilde{C}_{1}^{\chi 0}\right) \tilde{C}_{1}\right)^{\frac{1}{a}}\left(\left\|z_{\varepsilon}^{\frac{p}{2}}\right\|_{L^{1}(\Omega)}^{\frac{2(1-a)}{a}}\left\|\left(z_{\varepsilon}^{\frac{p}{2}}\right)_{X}\right\|_{L^{2}(\Omega)}^{2}+\left\|z_{\varepsilon}^{\frac{p}{2}}\right\|_{L^{1}(\Omega)}^{\frac{2}{a}}\right)\right]^{a}, t \geq t_{1},
\end{aligned}
$$


where $a=\frac{1+\chi}{3} \in\left(\frac{1}{3}, \frac{2}{3}\right)$ and $\tilde{C}_{1}$ is a fixed positive constant that only depends on $\chi_{0}$ because $\Omega$ is fixed and $\frac{2}{1-\chi} \in\left[2, \frac{2}{1-\chi_{0}}\right]$. By (72) and (69), we thus deduce that

$$
\begin{aligned}
\int_{\Omega}\left(z_{\varepsilon}^{\frac{p}{2}}\right)_{x}^{2} \geq & \left(2\left(1+\tilde{c}_{1}^{\chi_{0}}\right) \tilde{c}_{1}\right)^{-\frac{1}{a}} \cdot\left(\int_{\Omega} z_{\varepsilon}^{\frac{p}{2}}\right)^{-\frac{2(1-a)}{a}} \cdot\left(\int_{\Omega} v_{\varepsilon}^{\chi} z_{\varepsilon}^{p}\right)^{\frac{1}{a}}-\left(\int_{\Omega} z_{\varepsilon}^{\frac{p}{2}}\right)^{2} \\
\geq & \left(2\left(1+\tilde{c}_{1}^{\chi_{0}}\right) \tilde{c}_{1}\right)^{-\frac{1}{a}} \tilde{c}_{0}^{\frac{2(1-a)}{a}} \chi_{0} \cdot\left(\int_{\Omega} v_{\varepsilon}^{\chi} z_{\varepsilon}^{\frac{p}{2}}\right)^{-\frac{2(1-a)}{a}} \cdot\left(\int_{\Omega} v_{\varepsilon}^{\chi} z_{\varepsilon}^{p}\right)^{\frac{1}{a}} \\
& -\tilde{c}_{0}^{-2 \chi_{0}}\left(\int_{\Omega} v_{\varepsilon}^{\chi} z_{\varepsilon}^{\frac{p}{2}}\right)^{2}, t \geq t_{1} .
\end{aligned}
$$

Inserting this estimate into (71) and using once more (69), we obtain

$$
\begin{aligned}
\frac{\partial}{\partial t} \int_{\Omega} v_{\varepsilon}^{\chi} z_{\varepsilon}^{p} \leq & 1+\tilde{B}_{p} \int_{\Omega} v_{\varepsilon}^{\chi} z_{\varepsilon}^{p}-\tilde{D}_{p}\left(\int_{\Omega} v_{\varepsilon}^{\chi} z_{\varepsilon}^{\frac{p}{2}}\right)^{-\frac{2(1-a)}{a}} \cdot\left(\int_{\Omega} v_{\varepsilon}^{\chi} z_{\varepsilon}^{p}\right)^{\frac{1}{a}} \\
& +\tilde{E}_{p}\left(\int_{\Omega} v_{\varepsilon}^{\chi} z_{\varepsilon}^{\frac{p}{2}}\right)^{2}, \text { for } t \geq t_{1}
\end{aligned}
$$

where

$$
\begin{gathered}
\tilde{D}_{p}:=A_{p} \tilde{c}_{0}^{\chi_{0}}\left(2\left(1+\tilde{c}_{1}^{\chi_{0}}\right) \tilde{c}_{1}\right)^{-\frac{1}{a}} \tilde{c}_{0}^{\frac{2(1-a)}{a} \chi_{0}} \geq \frac{1}{2} \tilde{c}_{0}^{\chi_{0}}\left(2\left(1+\tilde{c}_{1}^{\chi_{0}}\right) \tilde{c}_{1}+1\right)^{-3} \tilde{c}_{0}^{4 \chi_{0}}=: D, \\
\tilde{E}_{p}:=A_{p} \tilde{c}_{0}^{\chi_{0}} \tilde{c}_{0}^{-2 \chi_{0}} \leq \tilde{c}_{0}^{-\chi_{0}}=: E, \tilde{B}_{p} \leq p^{2}(1+v)^{2}\left(1+\frac{(1+v)^{2}}{2 \tilde{c}_{0}^{\chi_{0}}}\right) \tilde{c}_{0}^{-\chi_{0}}+p^{2}=: b p^{2}
\end{gathered}
$$

for all $p \geq 2$, where $b, D$, and $E$ only depend on $\chi_{0}, v$, and $\lambda_{0}$.

Using again (66) and letting $\varepsilon \rightarrow 0$, we deduce like in the proof of Lemma 2.7 that

$$
\begin{aligned}
\frac{\partial}{\partial t} \int_{\Omega} v^{\chi} Z^{p} \leq & 1+b p^{2} \int_{\Omega} v^{\chi} Z^{p}-D\left(\int_{\Omega} v Z_{Z^{\frac{p}{2}}}\right)^{-\frac{2(1-a)}{a}} \cdot\left(\int_{\Omega} v^{\chi} Z^{p}\right)^{\frac{1}{a}} \\
& +E\left(\int_{\Omega} v^{\chi} Z^{\frac{p}{2}}\right)^{2}, \text { for } t \geq t_{1} .
\end{aligned}
$$

Next, we set $p_{k}:=2^{k}, k \in \mathbb{N}_{0}$, and

$$
y_{k}(t):=\int_{\Omega}\left(v^{x} z^{2^{k}}\right)(\cdot, t), t \geq t_{1}
$$

and obtain

$$
y_{k}^{\prime} \leq 1+b 2^{2 k} y_{k}-D y_{k-1}^{-\frac{2(1-a)}{a}} y_{k}^{\frac{1}{a}}+E y_{k-1}^{2}, t \geq t_{1}
$$

for all $k \geq 1$. Defining moreover

$$
\tau_{0}:=t_{1}, \tau_{k}:=\tau_{k-1}+b^{-1} 2^{-2 k}, \quad k \geq 1
$$

we set

$$
M_{k}:=\max \left\{1, \sup _{t \in\left[\tau_{k}, \infty\right)} y_{k}(t)\right\}, k \in \mathbb{N}_{0}
$$

and fixing $k \geq 1$, we conclude that

$$
y_{k}^{\prime} \leq 1+b 2^{2 k_{k}} y_{k}-D M_{k-1}^{-\frac{2(1-a)}{a}} y_{k}^{\frac{1}{a}}+E M_{k-1}^{2}, t \geq \tau_{k-1}
$$

Hence, if

$$
y_{k}(t) \geq M_{k-1}^{2} \cdot \max \left\{1,\left(\frac{4\left(1+b 2^{2 k}\right)}{D}\right)^{\frac{a}{1-a}},\left(\frac{4 E}{D}\right)^{a}\right\}=: \gamma_{k} M_{k-1}^{2}
$$

for $t \in[\alpha, \beta]$ with some $\tau_{k-1} \leq \alpha<\beta<\infty$, then we have

$$
\begin{aligned}
y_{k}^{\prime} & \leq\left(1+b 2^{2 k}\right) y_{k}^{-\frac{1-a}{a}} y_{k}^{\frac{1}{a}}-D M_{k-1}^{-\frac{2(1-a)}{a}} y_{k}^{\frac{1}{a}}+\frac{D}{4} M_{k-1}^{-\frac{2}{a}} y_{k}^{\frac{1}{a}} \cdot M_{k-1}^{2} \\
& \leq\left(\frac{D}{4}-D+\frac{D}{4}\right) M_{k-1}^{-\frac{2(1-a)}{a}} y_{k}^{\frac{1}{a}}=-\frac{D}{2} M_{k-1}^{-\frac{2(1-a)}{a}} y_{k}^{\frac{1}{a}}, t \in[\alpha, \beta] .
\end{aligned}
$$


Thus, an ODE comparison argument implies

$$
\begin{aligned}
y_{k}(t) & \leq\left(\frac{D(1-a)}{2 a} M_{k-1}^{-\frac{2(1-a)}{a}} \cdot(t-\alpha)+\left(y_{k}(\alpha)\right)^{-\frac{1-a}{a}}\right)^{-\frac{a}{1-a}} \\
& \leq \min \left\{y_{k}(\alpha),\left(\frac{D(1-a)}{2 a}\right)^{-\frac{a}{1-a}} M_{k-1}^{2} \cdot(t-\alpha)^{-\frac{a}{1-a}}\right\}, t \in[\alpha, \beta] .
\end{aligned}
$$

Hence, we have

$$
y_{k}(t) \leq\left(\gamma_{k}+1\right) M_{k-1}^{2} \text { for } t \geq \tau_{k-1}+\frac{2 a}{D(1-a)} \gamma_{k}^{-\frac{1-a}{a}}
$$

As $\gamma_{k} \geq\left(\frac{4 b 2^{2 k}}{D}\right)^{\frac{a}{1-a}}$ and $a \in\left(\frac{1}{3}, \frac{2}{3}\right)$, we obtain

$$
y_{k}(t) \leq\left(\gamma_{k}+1\right) M_{k-1}^{2} \text { for } t \geq \tau_{k}
$$

Hence, setting

$$
\gamma:=1+\left(\frac{4(1+b)}{D}\right)^{2}+\frac{4 E}{D}
$$

we deduce that

$$
M_{k} \leq\left(\gamma_{k}+1\right) M_{k-1}^{2} \leq \gamma 2^{4 k} M_{k-1}^{2}, \quad k \geq 1, M_{0} \leq 1+\tilde{k}_{1},
$$

where the latter is a consequence of (69) and $y_{0}=\int_{\Omega} u_{\varepsilon}$. We now proceed similar to the proof of Lemma 2.7 and conclude that

$$
Y_{k}:=\log _{2} M_{k} \leq 2^{k}\left(\log _{2}\left(1+\tilde{k}_{1}\right)+\sum_{j=1}^{\infty} \frac{4 j+\log _{2} \gamma}{2^{j}}\right)=: 2^{k} \tilde{S}
$$

$M_{k}^{\frac{1}{2^{k}}} \leq 2^{\tilde{s}}$ as well as

$$
\begin{aligned}
\sup _{t \in\left[t_{0}, \infty\right)}\|z\|_{L^{\infty}(\Omega)} & \leq \limsup _{k \rightarrow \infty} \sup _{t \in\left[t_{0}, \infty\right)}\|z\|_{L^{2^{k}}(\Omega)} \leq \limsup _{k \rightarrow \infty} \sup _{t \in\left[t_{0}, \infty\right)} c_{0}^{-\frac{x}{2^{k}}}\left|\int_{\Omega} v^{\chi} z^{2^{k}}\right|^{\frac{1}{2^{k}}} \\
& \leq \limsup _{k \rightarrow \infty} c_{0}^{-\frac{1}{2^{k}}} 2^{\tilde{S}}=2^{\tilde{S}}
\end{aligned}
$$

where $t_{0}:=t_{1}+\sum_{k=1}^{\infty} b^{-1} 2^{-2 k} \in(0, \infty)$, which satisfies $t_{0} \geq \tau_{k}$ for all $k \in \mathbb{N}_{0}$. Observe that in fact $\tilde{S}$ only depends on $\chi_{0}, \lambda_{0}$, and $\nu$, whereas $t_{0}$ additionally depends on $u_{0}$ and $v_{0}$, so that we have proved the claimed estimates because (68) is implied by (69) and Lemma 2.6 .

\section{Steady states}

In this section, we study the steady states $\left(u_{s}, v_{s}\right)$ of $(6)$ that are solutions of the problem

$$
\left\{\begin{array}{l}
-u_{s x x}=-\chi\left(\frac{u_{s}}{v_{s}} v_{s x}\right)_{x}-\lambda u_{s}, x \in(0,1), \\
0=1-v_{s}+u_{s}, x \in(0,1), \\
u_{s x}-\chi \frac{u_{s}}{v_{s}} v_{s x}=-v, x=0, u_{s x}-\chi \frac{u_{s}}{v_{s}} v_{s x}=0, x=1 .
\end{array}\right.
$$

In order to analyze these solutions, we introduce a corresponding weak formulation of the problem.

Definition 3.1

Let $\chi>0$. Then by a weak solution of (76), we mean a couple $\left(u_{s}, v_{s}\right)$ of functions

$$
u_{s} \in H^{1}(\Omega), v_{s} \in H^{1}(\Omega)
$$

such that $u_{s} \geq 0$ and $v_{s}=u_{s}+1>0$ a.e. in $\Omega$ satisfying the identities

$$
\int_{\Omega} u_{s X} \varphi_{X}=\chi \int_{\Omega} \frac{u_{s}}{1+u_{s}} u_{s X} \varphi_{X}+v \varphi(0)-\lambda \int_{\Omega} u_{s} \varphi \text { for any } \varphi \in H^{1}(\Omega) .
$$

The existence and uniqueness of the weak solution of $(76)$ is asserted in the following lemma. 
Lemma 3.2

For any $\chi \in(0,1)$, there exists a unique weak solution to the problem

$$
\left\{\begin{array}{l}
-u_{s x x}=-\left(\chi \frac{u_{s}}{1+u_{s}} u_{s x}\right)_{x}-\lambda u_{s}, x \in(0,1) \\
\left(1-\chi \frac{u_{s}(0)}{1+u_{s}(0)}\right) u_{s x}(0)=-v, u_{s x}(1)=0
\end{array}\right.
$$

Proof

Existence. In order to prepare a fixed point argument, we consider the set $A$ defined by

$$
A:=\left\{w \in L^{2}(\Omega) \quad \mid w \geq 0,\|w\|_{L^{2}(\Omega)} \leq \frac{v \sqrt{2}}{\min \{1-\chi, \lambda\}}\right\} .
$$

Notice that $A$ is a convex and closed subset of $L^{2}(\Omega)$. Moreover, we let $F: A \subset L^{2}(\Omega) \rightarrow L^{2}(\Omega)$ be defined by $F(\tilde{W})=W$, where $w$ is the solution to the problem

$$
\begin{aligned}
& -\left[a(x) w_{x}\right]_{x}+\lambda w=0 \quad \text { in } \Omega, \\
& a(0) w_{x}(0)=-v, \quad w_{x}(1)=0,
\end{aligned}
$$

for $a(x):=1-\chi \frac{\bar{w}}{1+\bar{w}}$. Because $F: A \subset L^{2}(\Omega) \rightarrow H^{1}(\Omega)$ is continuous and the embedding $H^{1}(\Omega) \hookrightarrow L^{2}(\Omega)$ is compact, we see that $F$ is a compact operator. For the reader's convenience, let us present some details of the proof of the step $F(A) \subset A$. We multiply the equation by $w$ and integrate by parts over $\Omega$ to obtain

$$
\int_{\Omega}\left(1-\chi \frac{\tilde{w}}{1+\tilde{w}}\right)\left|w_{X}\right|^{2}+\lambda \int_{\Omega} w^{2}=v w(0)
$$

that implies

$$
(1-\chi) \int_{\Omega}\left|w_{x}\right|^{2}+\lambda \int_{\Omega} w^{2} \leq v w(0)
$$

Because $|w(0)| \leq|w(x)|+\int_{0}^{x}\left|w_{x}\right|$, after an integration we have

$$
|w(0)| \leq\left|\int_{\Omega} w^{2}\right|^{\frac{1}{2}}+\left.\left.\left|\int_{\Omega}\right| w_{x}\right|^{2}\right|^{\frac{1}{2}} \leq\left.\left.\sqrt{2}\left|\int_{\Omega} w^{2}+\int_{\Omega}\right| w_{x}\right|^{2}\right|^{\frac{1}{2}} .
$$

In conjunction with (79), this proves that $F(A) \subset A$. Therefore, we may apply the Schauder fixed point theorem to obtain the existence of at least one fixed point $w \in A \cap H^{1}(\Omega)$, which clearly is a weak solution of (78).

Uniqueness. Let us write (78) in the form

$$
-\left((1-\chi) u_{s X}+\chi\left(\log \left(1+u_{s}\right)\right)_{X}\right)_{X}+\lambda u_{s}=0
$$

Letting $h$ be defined by

$$
h(s):=(1-\chi) s+\chi \log (1+s)
$$

and defining $w=h\left(u_{5}\right)$, we see that (78) becomes

$$
\left\{\begin{array}{c}
-w_{x x}=-\lambda h^{-1}(w) \\
w_{x}(0)=-v, w_{x}(1)=0 .
\end{array}\right.
$$

This entails uniqueness because $h^{-1}$ is a strictly increasing function.

Furthermore, we obtain a uniform bound on the spatial derivative of $u_{s}$.

Lemma 3.3

Let $\chi \in(0,1)$, and let $\left(u_{5}, v_{s}\right)$ denote the solution of (78). Then

$$
-\frac{v}{1-\chi} \leq u_{s x} \leq 0 \text { in } \bar{\Omega} .
$$

Proof

Rewriting (78) yields

$$
\left(1-\chi \frac{u_{s}}{1+u_{s}}\right) u_{s x x}=\lambda u_{s}+\frac{\left|u_{s x}\right|^{2}}{\left(1+u_{s}\right)^{2}} \text { in } \Omega
$$


As $u_{s} \geq 0$ and $\chi<1$, we therefore deduce that

$$
1-\chi \frac{u_{s}}{1+u_{s}}>0 \text { and } \lambda u_{s}+\frac{\left|u_{s x}\right|^{2}}{\left(1+u_{s}\right)^{2}} \geq 0,
$$

which gives

$$
u_{s x x} \geq 0
$$

Because, moreover, $u_{s x}(1)=0$, we have

$$
\min _{x \in \bar{\Omega}} u_{s x}(x)=u_{s x}(0)=-\frac{v}{1-\chi \frac{u_{s}(0)}{1+u_{s}(0)}} \geq-\frac{v}{1-\chi}
$$

and obtain (82).

\section{Stability of the steady state}

Finally, we show that the solution $(u, v)$ of $(6)$ converges to the unique steady state of $(6)$ as $t \rightarrow \infty$.

Theorem 4.1

Let $\lambda_{0}>0$ and $\chi_{0} \in(0,1)$, and assume that $\lambda \geq \lambda_{0}$ and $\chi \in\left(0, \chi_{0}\right]$ satisfy

$$
\chi<\frac{1}{2 C_{\infty}\left(\lambda_{0}, \chi_{0}\right)} \quad \text { and } \quad \lambda>\frac{4 \chi^{2} v^{2}}{(1-\chi)^{2}\left(1-2 \chi C_{\infty}\left(\lambda_{0}, \chi_{0}\right)\right)}
$$

where $C_{\infty}\left(\lambda_{0}, \chi_{0}\right)$ is as provided by Corollary 2.8 . Then for any choice of $\left(u_{0}, v_{0}\right)$ satisfying $(7)$, the global weak solution $(u, v)$ of $(6)$ obtained in Lemma 2.6 satisfies

$$
\lim _{t \rightarrow \infty} \int_{\Omega}\left|u-u_{s}\right|^{2}+\int_{\Omega}\left|v-v_{s}\right|^{2}+\int_{\Omega}\left|\left(v-v_{s}\right)_{X}\right|^{2}=0
$$

with exponential convergence rate. Here, $\left(u_{s}, v_{s}\right)$ with $v_{s}=u_{s}+1$ denotes the unique weak solution of the steady-state problem (76).

Proof

We write $(u, v)$ as a perturbation of the steady state, that is, we substitute $u=u_{s}+u_{1}, v=v_{s}+v_{1}$, where consequently $\left(u_{1}, v_{1}\right)$ satisfy

$$
\left\{\begin{array}{l}
u_{1 t}=u_{1 x x}-\chi\left(\frac{u_{1}}{v} v_{s x}+\frac{u}{v} v_{1 x}-\frac{u_{s} v_{1}}{v_{s} v} v_{s x}\right)_{x}-\lambda u_{1}, x \in(0,1), t>0 \\
v_{1 t}+v_{1}=u_{1}, x \in(0,1), t>0 \\
u_{1 x}-\chi\left(\frac{u_{1}}{v} v_{s x}+\frac{u}{v} v_{1 x}-\frac{u_{s} v_{1}}{v_{s} v} v_{s x}\right)=0, x \in\{0,1\}, t>0 \\
u_{1}(x, 0)=u_{10}(x), \quad v_{1}(x, 0)=v_{10}(x), x \in(0,1) .
\end{array}\right.
$$

We let $\delta$ be a positive number satisfying

$$
\frac{2 \chi^{2} v^{2}}{(1-\chi)^{2} \lambda}<\delta<\frac{1-2 C_{\infty} \chi}{2}
$$

We note that the assumption (84) guarantees the existence of such $\delta$. Next, we multiply the first equation in ( 85 ) by $u_{1}$ and integrate by parts to obtain

$$
\frac{\mathrm{d}}{\mathrm{d} t} \frac{1}{2} \int_{\Omega} u_{1}^{2}+\int_{\Omega}\left|u_{1 x}\right|^{2}=\chi \int_{\Omega}\left(\frac{u_{1}}{v} v_{s x} u_{1 x}+\frac{u}{v} v_{1 x} u_{1 x}-\frac{u_{s} v_{1}}{v_{s} v} v_{s x} u_{1 x}\right)-\lambda \int_{\Omega} u_{1}^{2}
$$

As $v_{s x}=u_{s x}$, we have $\left|v_{s x}\right| \leq \frac{v}{1-\chi}$ due to (82). We now only consider $t \geq t_{0}$, where $t_{0}$ is taken from Corollary 2.8 . Because $v \geq \frac{1}{2}$ by (68) and $\frac{u}{v^{x}} \leq C_{\infty}$ by (67) for $t \geq t_{0}$, we can estimate the terms on the right hand side of (87) according to

$$
\iota_{1}:=\chi \int_{\Omega} \frac{u_{1}}{v} v_{s x} u_{1 x} \leq \frac{\chi^{2} v^{2}}{4\left(\frac{1}{2}\right)^{2}(1-\chi)^{2} \delta} \int_{\Omega} u_{1}^{2}+\delta \int_{\Omega}\left|u_{1 x}\right|^{2}
$$

and

$$
I_{2}:=\chi \int_{\Omega} \frac{u}{v} v_{1 x} u_{1 x} \leq 2 C_{\infty} \chi\left(\frac{1}{2} \int_{\Omega}\left|v_{1 x}\right|^{2}+\frac{1}{2} \int_{\Omega}\left|u_{1 x}\right|^{2}\right)
$$


Now, we differentiate the second equation in (85) to obtain

$$
\frac{\partial}{\partial t} v_{1 x}+v_{1 x}=u_{1 x}
$$

in the natural weak sense, which implies

$$
\begin{aligned}
\int_{\Omega}\left|u_{1 x}\right|^{2} & =\int_{\Omega}\left(\frac{\partial}{\partial t} v_{1 x}+v_{1 x}\right)^{2}=\int_{\Omega}\left|\frac{\partial}{\partial t} v_{1 x}\right|^{2}+\int_{\Omega}\left|v_{1 x}\right|^{2}+\frac{\partial}{\partial t} \int_{\Omega}\left|v_{1 x}\right|^{2} \\
& \geq \int_{\Omega}\left|v_{1 x}\right|^{2}+\frac{\partial}{\partial t} \int_{\Omega}\left|v_{1 x}\right|^{2} .
\end{aligned}
$$

Therefore, (89) yields

$$
\iota_{2} \leq 2 C_{\infty} \chi\left(\int_{\Omega}\left|u_{1 x}\right|^{2}-\frac{\partial}{\partial t} \frac{1}{2} \int_{\Omega}\left|v_{1 x}\right|^{2}\right)
$$

Next, using $\frac{u_{s}}{v_{s}}=\frac{u_{s}}{u_{s}+1} \leq 1$ and $v_{s x}=u_{s x}$, we estimate

$$
J_{3}:=-\chi \int_{\Omega} \frac{u_{s} v_{1}}{v_{s} v} v_{s x} u_{1 X} \leq 2 \chi\left\|u_{s x}\right\|_{L \infty}(\Omega) \int_{\Omega}\left|v_{1}\right|\left|u_{1 X}\right| .
$$

Because the second equation in (85) implies

$$
\int_{\Omega} u_{1}^{2}=\int_{\Omega}\left|\frac{\partial}{\partial t} v_{1}+v_{1}\right|^{2}=\int_{\Omega}\left|\frac{\partial}{\partial t} v_{1}\right|^{2}+\int_{\Omega} v_{1}^{2}+\frac{\partial}{\partial t} \int_{\Omega} v_{1}^{2} \geq \int_{\Omega} v_{1}^{2}+\frac{\partial}{\partial t} \int_{\Omega} v_{1}^{2},
$$

we deduce by Lemma 3.3 that

$$
l_{3} \leq \frac{\chi^{2} v^{2}}{(1-\chi)^{2} \delta}\left(\int_{\Omega} u_{1}^{2}-\frac{\partial}{\partial t} \int_{\Omega} v_{1}^{2}\right)+\delta \int_{\Omega}\left|u_{1 x}\right|^{2}
$$

Setting

$$
a_{1}:=\frac{\chi^{2} v^{2}}{(1-\chi)^{2} \delta} \text { and } a_{2}:=C_{\infty} \chi
$$

we conclude by (87), (88), (91), and (93) that

$$
\frac{\mathrm{d}}{\mathrm{d} t}\left(\frac{1}{2} \int_{\Omega} u_{1}^{2}+a_{2} \int_{\Omega}\left|v_{1 x}\right|^{2}+a_{1} \int_{\Omega} v_{1}^{2}\right)+\left(1-2 a_{2}-2 \delta\right) \int_{\Omega}\left|u_{1 x}\right|^{2} \leq\left(-\lambda+2 a_{1}\right) \int_{\Omega} u_{1}^{2}
$$

for $t \geq t_{0}$. Hence, we have

$$
\frac{\mathrm{d}}{\mathrm{d} t}\left(\frac{1}{2} \int_{\Omega} u_{1}^{2}+a_{2} \int_{\Omega}\left|v_{1 x}\right|^{2}+a_{1} \int_{\Omega} v_{1}^{2}\right)+a_{4} \int_{\Omega}\left|u_{1 x}\right|^{2} \leq-2 a_{3} \int_{\Omega} u_{1}^{2}
$$

where

$$
a_{3}:=\frac{\lambda-2 a_{1}}{2} \text { and } a_{4}:=1-2 a_{2}-28
$$

are both positive due to (84) and (86). By (90) and (92), we finally deduce that

$$
\frac{\mathrm{d}}{\mathrm{d} t}\left(\frac{1}{2} \int_{\Omega} u_{1}^{2}+\left(a_{2}+a_{4}\right) \int_{\Omega}\left|v_{1 x}\right|^{2}+\left(a_{1}+a_{3}\right) \int_{\Omega} v_{1}^{2}\right) \leq-a_{3} \int_{\Omega} u_{1}^{2}-a_{3} \int_{\Omega} v_{1}^{2}-a_{4} \int_{\Omega}\left|v_{1 x}\right|^{2}
$$

for $t \geq t_{0}$. Therefore, Gronwall's lemma ends the proof.

\section{Numerical simulations}

In this section, we shall present some numerical experiments in order to illustrate the solution behavior in (6). The simulations were carried out using a time-explicit finite difference scheme on an equidistant spatial grid with grid size 0.005 and time step size $10^{-6}$. In all diagrams below, the ordinate and abscissa represent the spatial variable $x \in[0,1]$ and the time $t \geq 0$, respectively. Figure 1 underlines the attractivity result derived for the steady state of (6) in this paper when $\chi$ is "small" and $\lambda$ is "large". Because we did not derive explicit bounds for the constant $C_{\infty}\left(\chi_{0}, \lambda_{0}\right)$ introduced in Corollary 2.8 , we do not carry out a precise verification of (84) here. Nevertheless, the steady state seems to be attractive also for this choice of parameters.

Going beyond this, Figure 2 indicates that solutions of (6) still stabilize when neither of our sufficient conditions for the statements in Lemma 3.2 and Theorem 4.1 are satisfied.

Figure 3 shows the behavior of the solution in Figure 2 on a shorter time interval. It can be seen that a local maximum of $u$ appears in the interior of the spatial domain $(0,1)$ at some small time, and that this maximum slowly moves towards the origin. This indicates that on intermediate time scales, (6) might exhibit quite rich dynamical properties that have not been addressed by our analysis. 

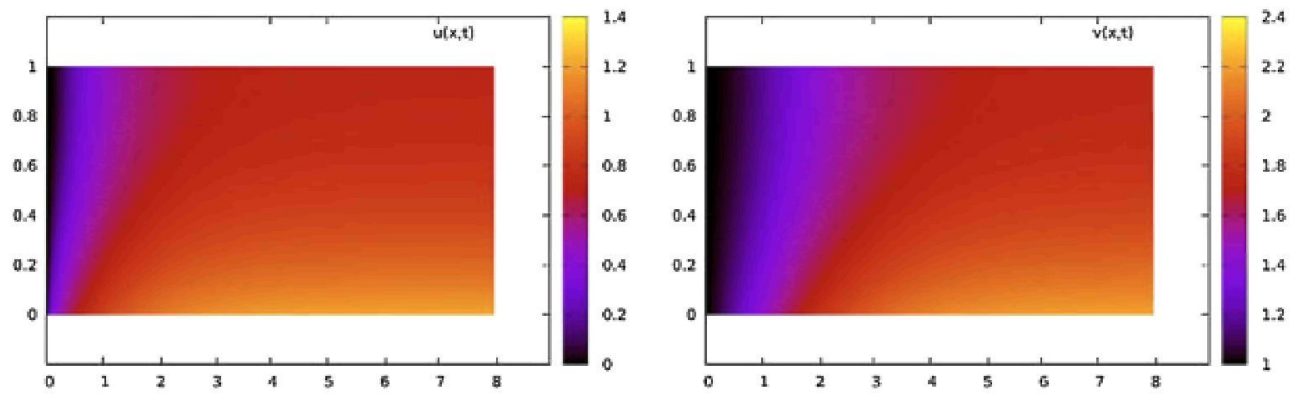

Figure 1. Solution behavior for $\chi=0.1, \lambda=1, \nu=1, u_{0} \equiv 0$ and $v_{0} \equiv 1$. Vertical axis: $x_{i}$ horizontal axis: $t$.
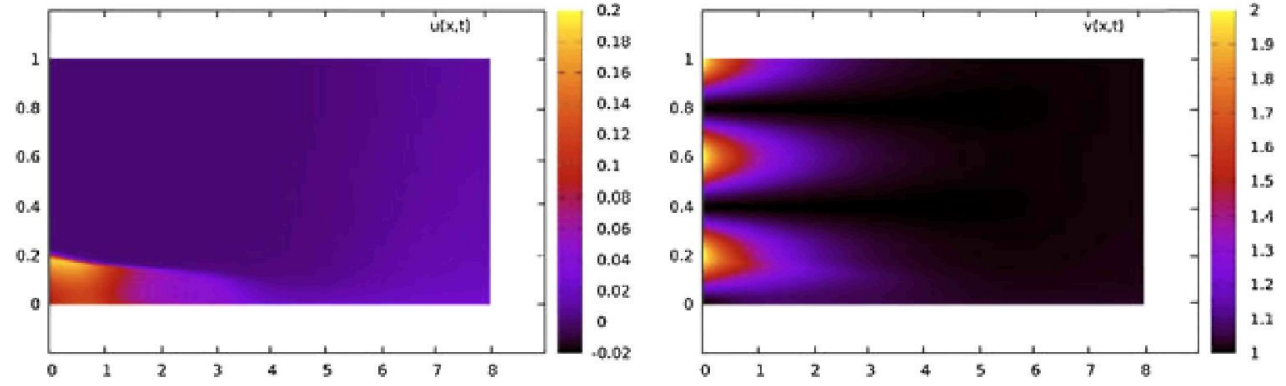

Figure 2. Large time behavior for $\chi=10, \lambda=1, v=1, u_{0} \equiv 0$ and $v_{0}(x)=1+\sin ^{2}(2.5 \pi x)$.
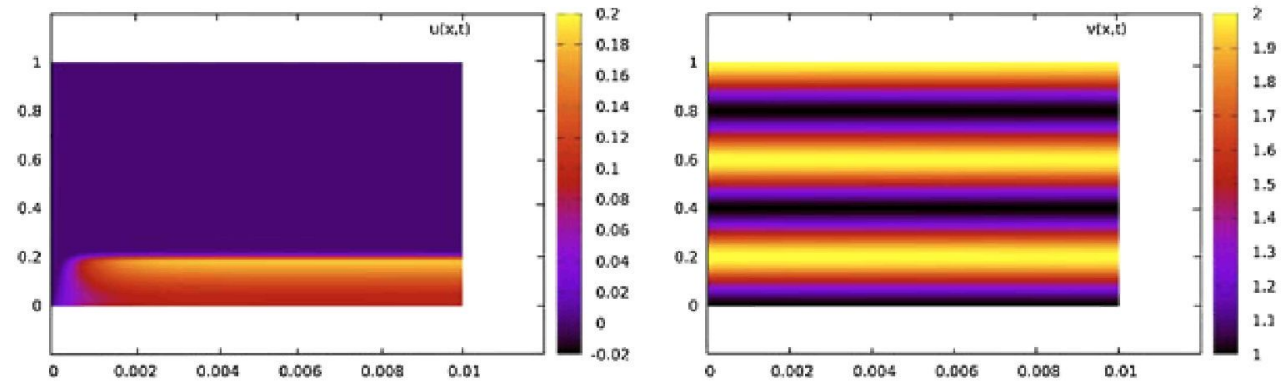

Figure 3. Behavior for $0 \leq t \leq 0.01$ for $\chi=10, \lambda=1, v=1, u_{0} \equiv 0$ and $v_{0}(x)=1+\sin ^{2}(2.5 \pi x)$.

\section{Acknowledgements}

This work was supported by the visiting professorship program Mercator of the Deutsche Forschungsgemeinschaft within which J.I. Tello visited the University of Duisburg-Essen in autumn 2010. The second author also acknowledges support by project MTM2009-13655 MICINN (Spain). The authors would like to thank Christian Thiel for his valuable assistance concerning the numerical simulations.

\section{References}

1. Entchev EV, Schwabedissen A, González-Gaitán M. Gradient formation of the TGF $\beta$ homolog Dpp. Cell 2000; 103:981-991.

2. Entchev EV, González-Gaitán M. Morphogen gradient formation and vesicular trafficking. Traffic 2002; 3:98-109.

3. Kerszberg M, Wolpert L. Mechanism for positional signalling by morphogen transport: A theoretical study. Journal of Theoretical Biology 1998; 191:103-114.

4. Krzyżanowski P, Laurençot Ph, Wrzosek D. Well-posedness and convergence to the steady state for a model of morphogen transport. SIAM Journal on Mathematical Analysis 2008; 40(5): 1725-1749.

5. Krzyżanowski P, Laurençot Ph, Wrzosek D. Mathematical models of receptor-mediated transport of morphogens. Mathematical Models and Methods in Applied Sciences 2010; 20(11):2021-2052.

6. Lander AD, Nie Q, Vargas B, Wan FYM. Aggregation of a distributed source morphogen gradient degradation. Studies in Applied Mathematics 2005; 114(4):343-374.

7. Lander AD, Nie Q, Wan FYM. Do morphogen gradients arise by diffusion? Developmental Cell 2002; 2:785-796.

8. Lander AD, Nie Q, Wan FYM. Internalization and end flux in morphogen gradient degradation. Journal of Computational and Applied Mathematics 2006; 190(1-2):232-251.

9. Turing AM. The chemical basis of morphogenesis. Philosophical Transactions of the Royal Society of London 1952; 237:37-72.

10. Bollenbach T, Kruse K, Pantazis P, González-Gaitán M, Jülicher F. Morphogen transport in epithelia. Physical Review E 2007; 75:011901. 


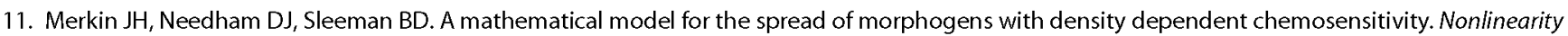
2005; 18(6):2745-2773.

12. Levine HA, Sleeman BD. A system of reaction-diffusion equations arising in the theory of reinforced random walks. SIAM Journal on Applied Mathematics 1997; 57(3):683-730.

13. Othmer HG, Stevens A. Aggregation, blowup, and collapse: The ABC's of taxis in reinforced random walks. SIAM Journal on Applied Mathematics 1997; 57(4):1044-1081.

14. Hillen T, Painter K. A user's guide to PDE models for chemotaxis. Journal of Mathematical Biology 2009; 58(1-2):183-217.

15. Winkler M. Global solutions in a fully parabolic chemotaxis system with singular sensitivity. Mathematical Methods in the Applied Sciences 2011 ; 34(2):176-190.

16. Winkler M. Absence of collapse in a parabolic chemotaxis system with signal-dependent sensitivity. Mathematische Nachrichten 2010; 283(11): 1664-1673.

17. Aida M, Osaki $K$, Tsujikawa T, Yagi A, Mimura M. Chemotaxis and growth system with singular sensitivity function. Nonlinear Analysis RWA 2005; 6(2):323-336.

18. Horstmann D, Winkler M. Boundedness vs. blow-up in a chemotaxis system. Journal of Differential Equations 2005; 215(1):52-107. 